\title{
Greenhouse Effect: The Relative Contributions of Emission Height and Total Absorption
}

\author{
JEAN-LOUIS DUFRESNE \\ Laboratoire de Météorologie Dynamique/IPSL, CNRS, Sorbonne Université, École Normale Supérieure, PSL Research \\ University, École Polytechnique, Paris, France \\ VINCENT EYMET \\ MesoStar, Toulouse, France \\ CYril CREVOISIER AND JEAN-Yves GRANDPEIX \\ Laboratoire de Météorologie Dynamique/IPSL, CNRS, École Polytechnique, Sorbonne Université, École Normale \\ Supérieure, PSL Research University, Paris, France
}

(Manuscript received 8 March 2019, in final form 22 November 2019)

\begin{abstract}
Since the 1970s, results from radiative transfer models unambiguously show that an increase in the carbon dioxide $\left(\mathrm{CO}_{2}\right)$ concentration leads to an increase of the greenhouse effect. However, this robust result is often misunderstood and often questioned. A common argument is that the $\mathrm{CO}_{2}$ greenhouse effect is saturated (i.e., does not increase) as $\mathrm{CO}_{2}$ absorption of an entire atmospheric column, named absorptivity, is saturated. This argument is erroneous first because absorptivity by $\mathrm{CO}_{2}$ is currently not fully saturated and still increases with $\mathrm{CO}_{2}$ concentration and second because a change in emission height explains why the greenhouse effect may increase even if the absorptivity is saturated. However, these explanations are only qualitative. In this article, we first propose a way of quantifying the effects of both the emission height and absorptivity and we illustrate which one of the two dominates for a suite of simple idealized atmospheres. Then, using a line-by-line model and a representative standard atmospheric profile, we show that the increase of the greenhouse effect resulting from an increase of $\mathrm{CO}_{2}$ from its current value is primarily due (about $90 \%$ ) to the change in emission height. For an increase of water vapor, the change in absorptivity plays a more important role (about $40 \%$ ) but the change in emission height still has the largest contribution (about 60\%).
\end{abstract}

\section{Introduction}

To establish the physical laws that govern the surface temperature of a planet, Fourier $(1824,1837)$ made the analogy between a vessel covered with plates of glass and Earth's surface covered by the atmosphere (Pierrehumbert 2004). Using this framework, Arrhenius (1896) made the first estimate of the greenhouse effect and of the sensitivity of the surface temperature to a change in carbon dioxide $\left(\mathrm{CO}_{2}\right)$ concentration of the atmosphere. His computation was based on a single layer model where the surface was covered by an isothermal atmosphere for which

Corresponding author: J.-L. Dufresne, jean-louis.dufresne@Imd. jussieu.fr the outgoing longwave flux at the top of the atmosphere (TOA) reads

$$
\bar{F}=\overline{\hat{\mathcal{T}}}_{s} \bar{B}\left(T_{s}\right)+\left(1-\overline{\hat{\mathcal{T}}}_{s}\right) \bar{B}\left(T_{a}\right),
$$

where $\bar{B}(T)$ is the blackbody emission (i.e., the StefanBoltzmann law) for a temperature $T$ and $\hat{\mathcal{T}}_{s}$ is the total broadband hemispheric transmissivity, that is, the transmissivity for radiation crossing the whole atmosphere, from its top to the surface, averaged over the longwave domain (overbars refer to variables averaged over the longwave domain) and over a hemisphere. As we assume scattering in the longwave domain is negligible, the broadband absorptivity of the atmosphere in the longwave domain is equal to $1-\overline{\hat{\mathcal{T}}}_{s}$ and is equal to the broadband emissivity of the atmosphere. Also, $T_{s}$ is the 

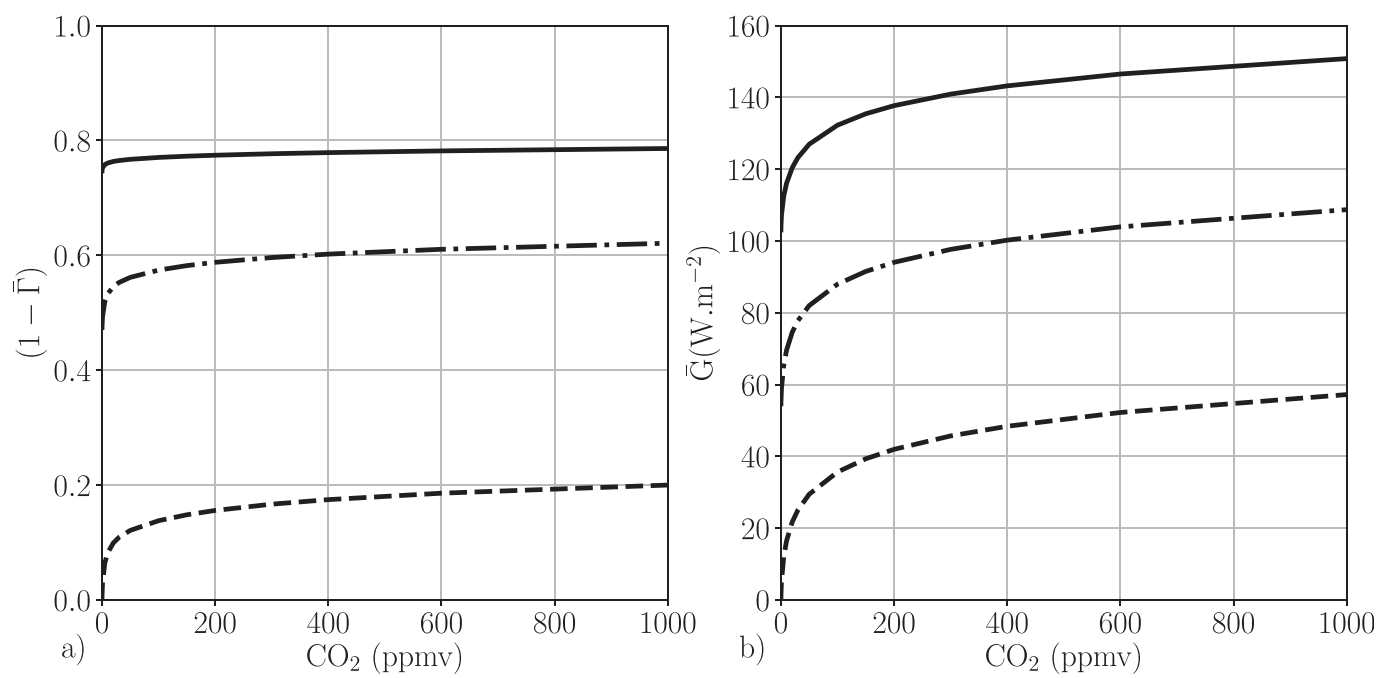

FIG. 1. (a) Broadband absorptivity $\left(1-\overline{\mathcal{T}}_{s}\right)$ of the atmosphere and (b) broadband greenhouse effect $\bar{G}$ at the tropopause as a function of the $\mathrm{CO}_{2}$ concentration for the standard MLS atmospheric profile (McClatchey et al. 1972; Anderson et al. 1986) (solid line), for the same profile where the water amount has been divided by 10 (dashdotted line) or set to zero (no water vapor; dashed line). $\mathrm{H}_{2} \mathrm{O}$ and $\mathrm{CO}_{2}$ are the only two absorbing gases considered. The computations have been done with the 4A line-by-line model (Scott and Chedin 1981; Cheruy et al. 1995). The broadband absorptivity is the average monochromatic absorptivity weighted by the Planck function at the surface temperature. The greenhouse effect at the tropopause is the difference between the flux emitted by the surface and the net flux at the tropopause $(200 \mathrm{hPa})$.

surface temperature and $T_{a}$ is a bulk temperature of the atmosphere, generally called emission temperature. The broadband greenhouse effect, defined as $\bar{G}=\bar{B}\left(T_{s}\right)-\bar{F}$, reads with this model as

$$
\bar{G}=\left(1-\overline{\hat{T}}_{s}\right)\left[\bar{B}\left(T_{s}\right)-\bar{B}\left(T_{a}\right)\right] .
$$

Although this equation has important limitations, it shows that the greenhouse effect is the product of two terms. The first is an optical characteristic, namely the absorptivity of the atmosphere $\left(1-\overline{\hat{\mathcal{T}}}_{s}\right)$. The larger the absorptivity is, the larger the greenhouse effect will be. The second is an energy term that depends on thermodynamic variables, the surface temperature, and the emission temperature of the atmosphere. The larger the difference between the two temperatures is, the larger the greenhouse effect will be.

The broadband absorptivity of the atmosphere increases when the amount of water vapor increases, which supports the simple idea that an increase of atmospheric absorptivity in the infrared increases the greenhouse effect. However, the broadband absorptivity shows very little increase when the $\mathrm{CO}_{2}$ concentration increases, especially for regular amounts of water vapor (Fig. 1a). This is the well-known "saturation effect" of $\mathrm{CO}_{2}$ absorption (Archer 2011; Pierrehumbert 2011; Zhong and Haigh 2013), first pointed out by Ångström (1900), who questioned the results of Arrhenius (1896) showing the impact of $\mathrm{CO}_{2}$ concentration on the Earth surface temperature. It has been shown that the $\mathrm{CO}_{2}$ absorption is not fully saturated (Pierrehumbert 2011; Shine et al. 1995), and that a $\mathrm{CO}_{2}$ increase modifies both the broadband and the spectral flux at the TOA (Kiehl 1983; Charlock 1984; Harries et al. 2001; Mlynczak et al. 2016). This saturation argument is still used in the public debate to claim that an increase of $\mathrm{CO}_{2}$ concentration has very limited impact, if any, on the greenhouse effect.

The "saturation paradox" can be summarized as follows: why does the greenhouse effect increase with the $\mathrm{CO}_{2}$ concentration (Fig. 1b) whereas the broadband absorptivity does not increase as much, especially when water vapor is present (Fig. 1a)? As highlighted by Eq. (2), the absorptivity is not the only main parameter that controls the greenhouse effect; the emission temperature $T_{a}$ of the atmosphere is also fundamental. If the increase of $\mathrm{CO}_{2}$ concentration has little impact on absorptivity, it has a significant impact on $T_{a}$. When the $\mathrm{CO}_{2}$ increases, the infrared radiation that escapes toward space is emitted by the atmosphere at a higher altitude. As most of the radiation is emitted by the troposphere, higher altitude means lower emission temperature, a lower value of the Planck function, a lower value of the radiation emitted toward space, and therefore a higher value of the greenhouse effect (Hansen et al. 1981; Pierrehumbert 2010; Archer 2011; Benestad 2017). For a doubling of the $\mathrm{CO}_{2}$ concentration, the average value of the 
change in emission height is about $150 \mathrm{~m}$, assuming that the radiative forcing of about $\approx 4 \mathrm{~W} \mathrm{~m}^{-2}$ can be translated into a change in blackbody temperature emission, and then into a change in emission height assuming a temperature vertical gradient of $\approx 6.5 \mathrm{~K} \mathrm{~km}^{-1}$ (Held and Soden 2000).

Beyond the single layer model, for fundamental physical reasons, the increase of the greenhouse effect due to an increase of the concentration of an absorbing gas, in particular $\mathrm{CO}_{2}$, is partly due to an increase of absorptivity and partly due to an increase of emission height (Pierrehumbert 2010). However, the contributions of each of these two effects have not been quantified yet, and the main goals of this paper are to present a framework that allows quantifying the contribution of these two effects and to perform the quantification. A second goal is to quantify the change in emission height, and not only its impact on the flux at the TOA. This offers the possibility to propose a new quantitative simplified description of the greenhouse effect that is more realistic than the too simple single layer model called the "blanket model" (Benestad 2017).

In this study, we will use only prescribed atmospheric profiles and will therefore compute the forcing when changing the absorbing gas concentration. All calculations are for cloudless skies. In section 2 we present the framework that allows us to separate and quantify the contribution of absorptivity and that of emission height to the flux at the tropopause, and therefore to the greenhouse effect. To allow some analytical developments, especially for simple limiting cases, we consider monochromatic radiances and idealized vertical atmospheric profiles. In section 3 we still consider radiances but with realistic atmospheric profiles. This will help us to interpret the results presented in section 4, where we compute the flux at the tropopause over the whole thermal infrared domain and where we independently increase the concentration of the two most important greenhouse gases on Earth: water vapor $\left(\mathrm{H}_{2} \mathrm{O}\right)$ and $\mathrm{CO}_{2}$. The temperature adjustment of the stratosphere is also analyzed. A summary and conclusions are given in section 5 .

\section{Formulation with simplified conditions}

To present the main concepts and to facilitate some analytical developments, we first consider the very simple case of an idealized atmosphere where the monochromatic absorption coefficient is constant along the vertical and the volumetric mass density only depends on pressure and therefore on altitude $z$ :

$$
\rho(z)=\rho(0) e^{-z / h_{r}},
$$

where $h_{r}$ is the scale height $\left(h_{r} \approx 8 \mathrm{~km}\right.$ on Earth). Hydrostatic pressure follows a law of the same type as density:

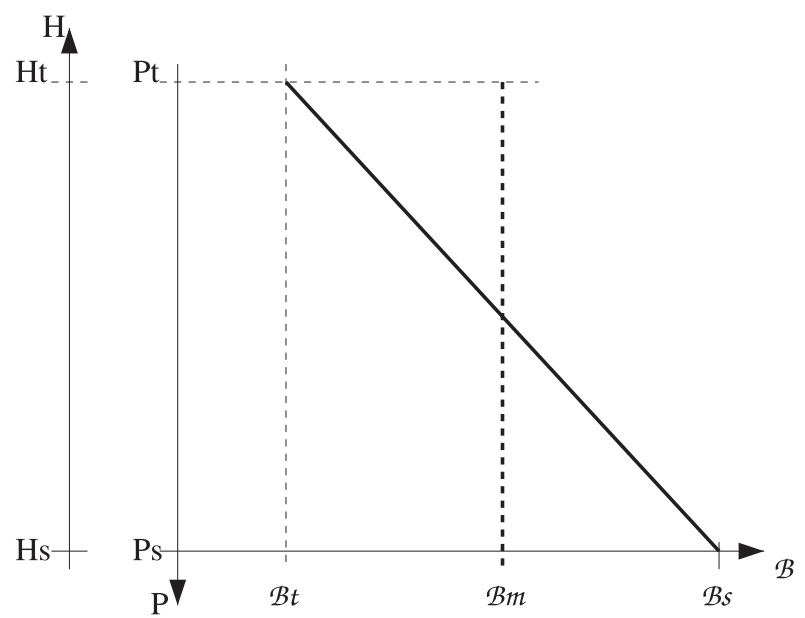

FIG. 2. Vertical profile of the Planck function $\mathcal{B}$ for an isothermal atmosphere (thick vertical dashed line at $\mathcal{B}_{m}$ ) and for an idealized temperature profile for which the Planck function increases linearly with pressure $P$ from $\mathcal{B}_{t}$ at the tropopause (pressure $P_{t}$ and altitude $H_{t}$ ) to $\mathcal{B}_{s}$ at the surface (pressure $P_{s}$ and altitude $H_{s}$ ) (thick vertical solid line). The pressure axis has a linear scale, whereas the altitude axis has a logarithmic scale.

$$
P(z)=P(0) e^{-z / h_{r}} .
$$

To quantify the radiative forcing of $\mathrm{CO}_{2}$, it has been shown that the change of the net flux at the tropopause is much more relevant than the change of the net flux at the top of the atmosphere (Shine et al. 1995; Hansen et al. 1997; Stuber et al. 2001). To keep the atmospheric profile as simple as possible, we ignore the stratosphere in a first step and then show section $4 \mathrm{~d}$ that this simplification has little impact for the key points addressed in this study. We therefore consider the troposphere only, that is, an atmosphere whose vertical extent ends at the tropopause. A last simplification is to assume that the temperature vertical profile is such that the monochromatic radiance emitted by a blackbody (or Planck function) $\mathcal{B}(P)$ increases linearly with pressure $P$ (Fig. 2):

$$
\mathcal{B}(P)=\mathcal{B}\left(P_{s}\right)+\frac{P-P_{s}}{P_{t}-P_{s}}\left[\mathcal{B}\left(P_{t}\right)-\mathcal{B}\left(P_{s}\right)\right],
$$

where $P_{s}=1000 \mathrm{hPa}$ and $P_{t}=200 \mathrm{hPa}$ are the pressure at the surface and at the tropopause, $H_{s}=0$ and $H_{t}=$ $h_{r} \log \left(P_{s} / P_{t}\right) \approx 12.9 \mathrm{~km}$ are the altitude of the surface and the tropopause, respectively. Note that curly letters refer to monochromatic directional variables. We consider two contrasted profiles (Fig. 2): a profile in which $\mathcal{B}$ decreases from the Planck function at surface $\mathcal{B}_{s}$ to a value $\mathcal{B}_{t}$ at the tropopause $\left[\mathcal{B}\left(P_{s}\right)=\mathcal{B}_{s}\right.$ and $\left.\mathcal{B}\left(P_{t}\right)=\mathcal{B}_{t}\right]$, and an isothermal profile chosen so that the two profiles have the same mass 
weighted mean value: $\mathcal{B}\left(P_{s}\right)=\mathcal{B}\left(P_{t}\right)=\mathcal{B}_{m}=0.5\left(\mathcal{B}_{t}+\mathcal{B}_{s}\right)$. The Planck function is computed for a wavenumber $\nu_{c}=$ $550 \mathrm{~cm}^{-1}$ (corresponding to a wavelength $\lambda_{c}=18 \mu \mathrm{m}$ ) close to the strong $\mathrm{CO}_{2} 15-\mu \mathrm{m}$ absorption band and for temperatures $T_{s}=294 \mathrm{~K}\left(\mathcal{B}_{s} \approx 0.144 \mathrm{~W} \mathrm{~m}^{-2} \mathrm{sr}^{-1}\right)$ and $T_{t}=220 \mathrm{~K}$ $\left(\mathcal{B}_{t} \approx 0.056 \mathrm{~W} \mathrm{~m}^{-2} \mathrm{sr}^{-1}\right)$. We assume that the atmosphere has a homogeneous concentration of absorbing gases and we neglect the effects of pressure and temperature on the specific absorption coefficient $k\left(\mathrm{~m}^{2} \mathrm{~kg}^{-1}\right)$. Therefore $k$ is constant along the vertical. The surface is assumed to be a perfect blackbody. We also assume that radiation propagates only along the vertical, which allows us to replace the integral on the zenith angle by considering one single angle. The radiative exchanges are computed at a given frequency and with a formalism adapted for general plane parallel atmospheres (Schwarzkopf and Fels 1991).

\section{a. Basic equations and the limiting case of the single layer model}

With the above assumptions, the expression of the optical thickness between the tropopause and a layer of pressure $P$ at altitude $z$ simplifies as

$$
\tau(P)=k f\left(P-P_{t}\right) / g,
$$

where $g$ is the gravity $\left(\mathrm{m} \mathrm{s}^{-2}\right)$. We introduce $f$, which is a multiplicative factor to allow a proportional change in absorption within the whole atmosphere. By default, $f=1$. The spectral outgoing radiance $\mathscr{F}$ at the tropopause in the zenith direction then reads (Pierrehumbert 2010):

$$
\begin{aligned}
\mathscr{F} & =\mathcal{T}_{s} \mathcal{B}_{s}+\int_{P_{t}}^{P_{s}} \frac{\partial \mathcal{T}(P)}{\partial P} \mathcal{B}(P) d P \\
& =\mathscr{F}_{s}+\mathscr{F}_{a}
\end{aligned}
$$

where $\mathcal{T}(P)$ is the directional transmissivity between altitude of pressure $P$ and the tropopause

$$
\mathcal{T}(P)=e^{-\tau(P)} ;
$$

$\mathcal{T}_{s}=\mathcal{T}\left(P_{s}\right)=e^{-\tau_{s}}$ is the transmissivity of the troposphere with $\tau_{s}=\tau\left(P_{s}\right)$ being the total optical thickness of the troposphere (i.e., from the tropopause to the surface); $\mathscr{\mathscr { F }}_{s}=\mathcal{T}_{s} \mathcal{B}_{s}$ is the radiance that is emitted by the surface and that reaches the tropopause, and $\mathscr{F}_{a}$ is the vertical integral of the radiance that is emitted by the troposphere and that reaches the tropopause.

Equation (6) may be written as

$$
\mathscr{F}=\mathcal{T}_{s} \mathcal{B}_{s}+\left(1-\mathcal{T}_{s}\right) \mathcal{B}_{e},
$$

where $\mathcal{B}_{e}$ is the equivalent blackbody emission of the atmosphere:

$$
\mathcal{B}_{e}=\int_{P_{t}}^{P_{s}} \mathcal{B}(P) \omega(P) d P
$$

and

$$
\omega(P)=\frac{1}{1-\mathcal{T}_{s}} \frac{\partial \mathcal{T}(P)}{\partial P} .
$$

Equation (8a) has the same form as the classical single layer model [Eq. (1)] except that here it is spectrally resolved. This equation makes explicit that the radiance at the tropopause depends directly on the transmissivity $\mathcal{T}_{s}$, and therefore on the total optical thickness $\tau_{s}$ of the troposphere. This transmissivity $\mathcal{T}_{s}$ impacts both the radiance that reaches the tropopause emitted by the surface and the radiance that reaches the tropopause emitted by the troposphere.

The equivalent blackbody emission $\mathcal{B}_{e}$ of the atmosphere is the mean value of the blackbody emission $\mathcal{B}(P)$ weighted by the $\omega(P)$ function [Eq. (8b)]. For optically very thin atmospheres $\left(\tau_{s} \ll 1\right), \mathcal{B}_{e}$ is equal to the pressure-weighted mean of $\mathcal{B}(P)$. Then $\mathcal{B}_{e} \approx\left[\mathcal{B}\left(P_{t}\right)+\right.$ $\left.\mathcal{B}\left(P_{s}\right)\right] / 2$ and is the same for the two idealized atmospheric profiles considered in this section (appendix A).

When the troposphere is optically thin, the radiance $\mathscr{\mathscr { T }}$ at the tropopause decreases when the optical thickness $\tau_{s}$ of the troposphere increases, starting from a value $\mathscr{F}=\mathcal{B}_{s}$ when $\tau_{s}=0$ (Fig. 3, black line). As long as $\tau_{s} \ll 1$, the decrease of the radiance $\mathscr{F}$ at the tropopause is proportional to $\tau_{s}$ and similar for both atmospheric profiles because the pressure-weighted mean temperature of both tropospheres is the same (see section a in appendix A). When the troposphere is isothermal, this decrease gradually slows down from optical thickness $\tau_{s}$ larger than 0.5 and reaches a plateau when the optical thickness is larger than about 4 . When the troposphere is nonisothermal, the slowdown is not as fast as for the isothermal case and the decrease continues for optical thickness larger than 4 . The limiting value of the radiance at the tropopause for infinite value of the optical thickness is much smaller (and therefore the greenhouse effect much higher) in the nonisothermal case compared to the isothermal case.

The model generally used in simplified explanations of the greenhouse effect [Eq. (1)] assumes that the troposphere is isothermal along the vertical. With this assumption, the flux at the tropopause does not decrease any more when the total optical thickness $\tau_{s}$ increases if $\tau_{s}$ is larger than 4 . It is then said that the greenhouse effect "saturates." This saturation effect almost disappears when the temperature decreases with 


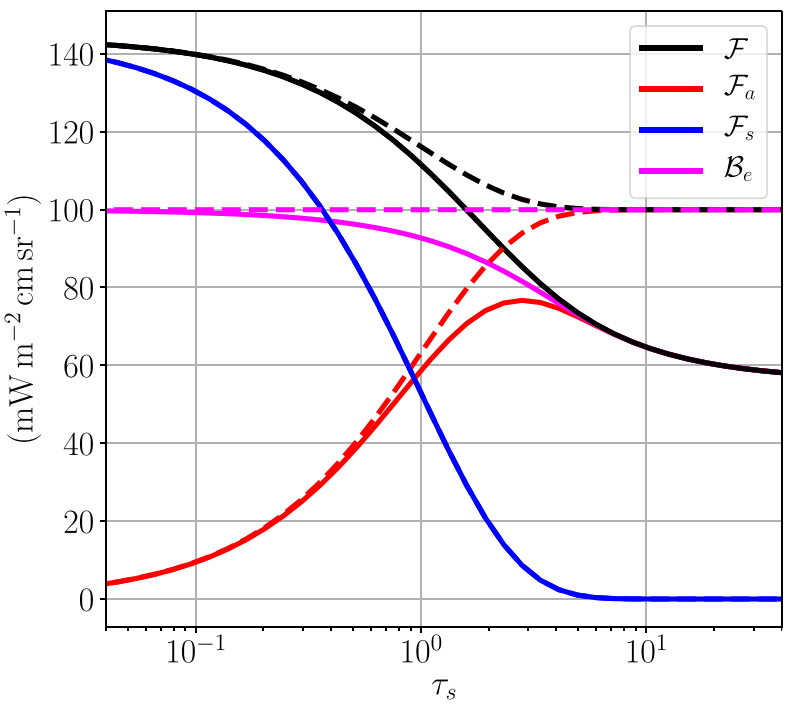

FIG. 3. Radiance at the tropopause [black: total, $\mathscr{F}$; blue: emitted by the surface, $\mathscr{F}_{s}$; red: emitted by the troposphere, $\mathscr{F}_{a}$; Eq. (6)], weighted average blackbody emission of the troposphere [magenta: $\mathcal{B}_{e}$; Eq. (8b)], as a function of the total optical thickness $\tau_{s}$ of the troposphere for an isothermal vertical profile (dashed line) and a profile for which the temperature decreases with altitude (solid line). The idealized troposphere is $12.6 \mathrm{~km}$ high with uniform absorption coefficient and volumetric mass density that only depends on pressure (see section $2 \mathrm{a}$ ).

height: the greenhouse effect continues to increase when the optical thickness $\tau_{s}$ increases, even for large values of $\tau_{s}$. For a nonisothermal troposphere the altitude where the emitted radiation escapes to space matters. We now present how this effect of emission height can be quantified.

\section{b. Contribution of absorptivity and emission height to radiance changes}

The sensitivity of the radiance $\mathscr{F}$ at the tropopause to a fractional change in amount of absorbing gases reads, according to Eq. (8):

$$
\frac{\partial \mathscr{\mathscr { F }}}{\partial f} \equiv \mathscr{\mathscr { F }}^{\prime}=\frac{\partial \mathcal{T}_{s}}{\partial f}\left(\mathcal{B}_{s}-\mathcal{B}_{e}\right)+\left(1-\mathcal{T}_{s}\right) \frac{\partial \mathcal{B}_{e}}{\partial f},
$$

which we rewrite as

$$
\begin{aligned}
\mathscr{F}^{\prime} & =\mathscr{\mathscr { F }}_{\mathcal{T}}^{\prime}+\widetilde{\mathscr{F}}_{Z_{e}}^{\prime}, \\
\mathscr{F}_{\mathcal{T}}^{\prime} & =\frac{\partial \mathcal{T}_{s}}{\partial f}\left(\mathcal{B}_{s}-\mathcal{B}_{e}\right), \quad \text { and } \\
\tilde{F}_{Z_{e}}^{\prime} & =\left(1-\mathcal{T}_{s}\right) \frac{\partial \mathcal{B}_{e}}{\partial f} .
\end{aligned}
$$

These three terms are shown in Fig. 4 as a function of the total optical thickness $\tau_{s}$ of the troposphere.

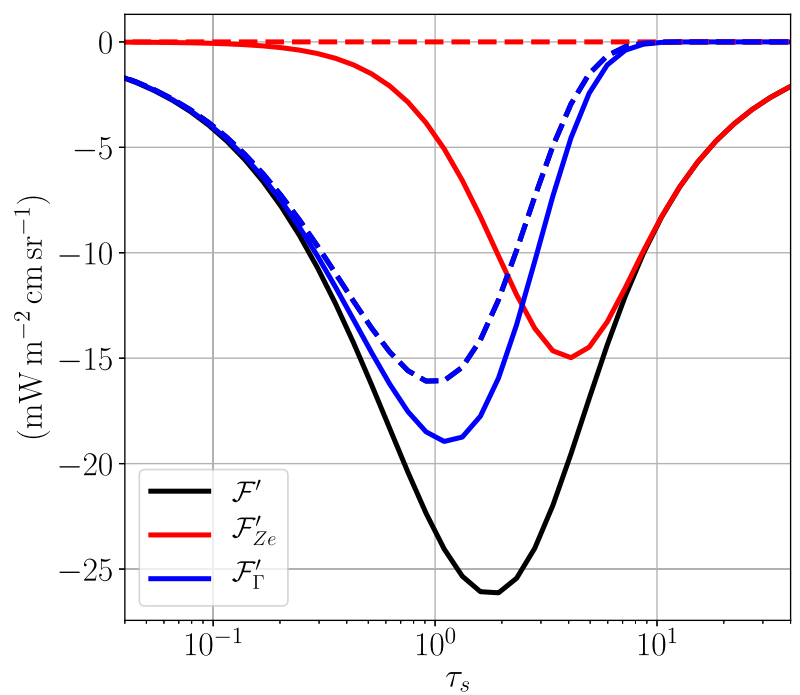

FIG. 4. Sensitivity $\mathscr{F}^{\prime}$ of the radiance at the tropopause to a fractional change in absorbing gas concentration as a function of the total optical thickness $\tau_{s}$ of the troposphere. Results are shown for the same isothermal (dashed line) and idealized decreasing temperature (solid line) profiles as in Fig. 3. The sensitivity $\mathscr{F}^{\prime}$ (black) is decomposed in a contribution due to change in absorptivity $\left(\mathscr{F}_{T}^{\prime}\right.$; blue line) and a contribution due to change in emission height $\left(\mathscr{F}_{Z_{e}}^{\prime}\right.$; red line). For the isothermal profile, $\mathscr{F}^{\prime}$ is not visible because it is covered by $\mathscr{F}_{\mathcal{T}}^{\prime}$.

According to Eq. (10b), $\mathscr{F}_{\mathcal{T}}^{\prime}$ quantifies how much the radiance $\mathscr{F}$ at the tropopause is directly impacted by a change in the transmissivity $\mathcal{T}_{s}$, and therefore by a change in the absorptivity $\mathcal{A}_{s}=1-\mathcal{T}_{s}$, when the amount of absorbing gases changes. The term $\mathscr{F}_{\mathcal{T}}^{\prime}$ is the sensitivity of the radiance at the tropopause if the troposphere is isothermal, or would be isothermal, at a temperature that corresponds to a blackbody emission $\mathcal{B}_{e}$ (i.e., $\partial \mathcal{B}_{e} / \partial f=0$ ). For both temperature profiles, the absolute value of $\mathscr{\mathscr { F }}_{\mathcal{T}}^{\prime}$ linearly increases with $\tau_{s}$, is maximum for $\tau_{s} \approx 1$, becomes very small for $\tau_{s}$ larger than 4 , and is almost zero when the troposphere is fully opaque (Fig. 4). Note that $\mathscr{F}_{\mathcal{T}}^{\prime}$ is slightly higher for the nonisothermal profile because $\mathcal{B}_{e}$ has a smallest value with this profile as compared with the isothermal profile (Fig. 3).

The term $\mathscr{F}_{Z_{e}}^{\prime}$ quantifies how much the radiance at the tropopause is impacted by a change in $\mathcal{B}_{e}$ when the amount of absorbing gases changes. Radiance $\mathcal{B}_{e}$ [Eq. (8b)] is the weighted average of the Planck function over the whole troposphere with a weight $\omega(P)$ [Eq. $(8 c)]$, which depends on the optical exchange factor between the atmosphere at pressure $P$ and the tropopause (Dufresne et al. 2005). This weight varies from a function that is constant with pressure when the total optical thickness is low $\left(\tau_{s} \ll 1\right)$ to a function that is maximum at the tropopause, decreases with increasing 


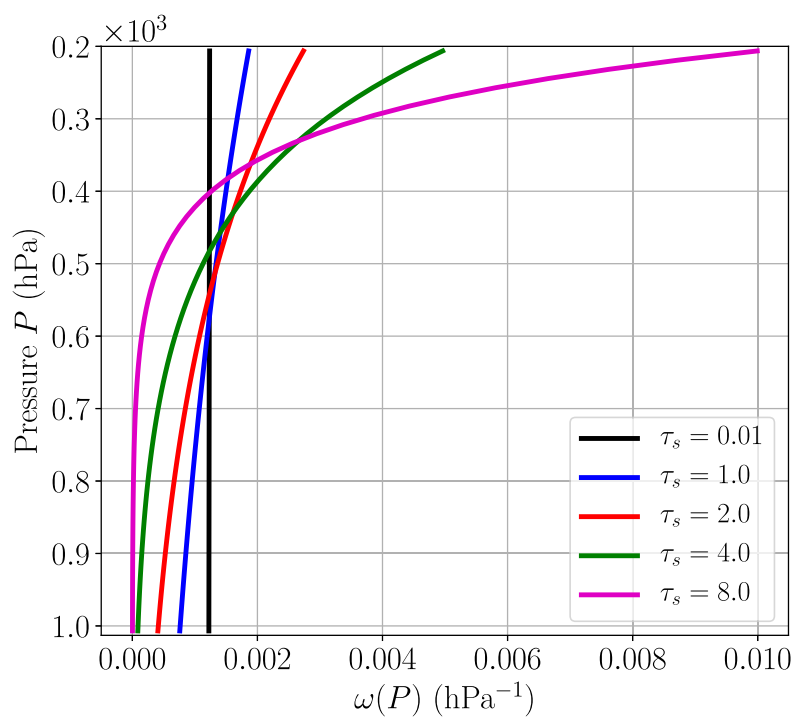

FIG. 5. Vertical profile of $\omega(P)$ for different values of the total optical thickness $\tau_{s}$ of the troposphere (black: 0.01, blue: 1, red: 2 , green: 4 , and magenta: 8 ) for the same idealized troposphere with a uniform absorption coefficient as in Fig. $3 ; \omega(P)$ is the normalized optical exchange factor between the troposphere at pressure $P$ and the tropopause [Eq. $(8 \mathrm{c})]$.

pressure and is almost zero close to the surface when the total optical thickness is large $\left(\tau_{s} \gg ; 1\right)$ (Fig. 5; section $\mathrm{b}$ in appendix $\mathrm{A})$. As a consequence, the radiation that reaches the tropopause is emitted on average at lower pressure (i.e., at higher altitude) when the optical thickness of the troposphere increases. It is said that the "emission height" increases (Hansen et al. 1981; Held and Soden 2000; Pierrehumbert 2010; Archer 2011; Benestad 2017). The variable $\tilde{\mathscr{F}}_{Z_{e}}^{\prime}$ quantifies how much this change in emission height impacts the radiance at the tropopause. It is zero for an isothermal troposphere since $\left(\partial \mathcal{B}_{e} / \partial f\right)=0$. If the temperature of the troposphere decreases with height, an increase of emission height yields a decrease of the temperature, a decrease of the Planck function and therefore a decrease of the upward radiance at the tropopause. The sensitivity $\mathscr{F}^{\prime}{ }_{Z_{e}}$ due to change in emission height increases with the total optical thickness $\tau_{s}$ of the troposphere, reaches a maximum for $\tau_{s} \approx 4$, and then slowly decreases (red line in Fig. 4).

\section{c. Emission height}

After defining the contribution of the change in emission height to the change in radiance at the tropopause, we now define the emission height itself. Since we assume in this section that the absorption coefficient $k$ is constant, the optical thickness increases linearly with pressure [Eq. (5)]. Therefore, many radiative variables are easier to compute and to interpret in pressure coordinate rather than in altitude coordinate. We will therefore continue to write the equations in pressure coordinate, and the "emission height" will be defined as the altitude corresponding to the "emission pressure."

The relative contribution $\Omega(P)$ of a layer of thickness $d P$ at pressure $P$ to the radiance $\mathscr{\mathscr { F }}_{a}$ reads, according to Eq. (6):

$$
\Omega(P) d P=\frac{\frac{\partial \mathcal{T}(P)}{\partial P} \mathcal{B}(P) d P}{\int_{P_{t}}^{P_{s}} \frac{\partial \mathcal{T}(P)}{\partial P} \mathcal{B}(P) d P} .
$$

Using Eqs. (8b) and (8c), this equation may be written as

$$
\begin{aligned}
\Omega(P) & =\frac{1}{\left(1-\mathcal{T}_{s}\right) \mathcal{B}_{e}} \frac{\partial \mathcal{T}(P)}{\partial P} \mathcal{B}(P) \\
& =\omega(P) \frac{\mathcal{B}(P)}{\mathcal{B}_{e}}
\end{aligned}
$$

According to Eq. (8b), $\int_{P_{t}}^{P_{s}} \Omega(P) d P=1, \Omega(P)$ is the probability density function that photons emitted by the troposphere and that reached the tropopause have been emitted at an altitude where the pressure is $P$. Therefore, the mean pressure where the photons reaching the tropopause have been emitted is

$$
P_{e}=\int_{P_{t}}^{P_{s}} P \Omega(P) d P
$$

This mean emission pressure $P_{e}$ will be simply named emission pressure and the emission height $Z_{e}$ will be defined as the altitude at which the pressure is equal to $P_{e}$. However, one should have in mind that the actual emission pressure and emission height are not single values but are functions that are nonzero in a wide pressure and altitude range. In particular they span the whole troposphere when the optical thickness is small.

When the troposphere is isothermal, $\mathcal{B}(P)=\mathcal{B}_{e}$ and therefore $\Omega(P)=\omega(P)$. The probability density that a photon emitted by the troposphere and that reached the tropopause has been emitted at a level of pressure $P$ is equal to the probability density that a photon going downward at the tropopause is absorbed at level of pressure $P$. This is consistent with the reciprocity principle. Therefore, when the total optical thickness $\tau_{s}$ is small, the emission pressure is equal to the average between the tropopause pressure and the pressure at surface, that is, $\left(P_{t}+P_{s}\right) / 2$. The corresponding altitude is slightly higher than $4 \mathrm{~km}$, which is consistent with what is observed in Fig. 6. Relative to the isothermal profile, $Z_{e}$ is lower when the temperature decreases with height because the Planck function gives more weight to the lower and warmer part of the troposphere. 


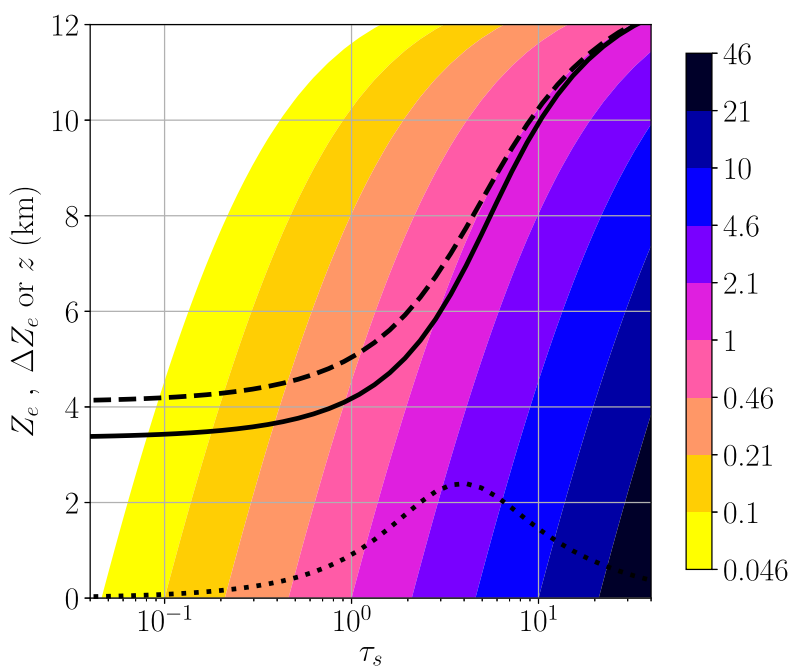

FIG. 6. Emission height $Z_{e}$ (black lines) as a function of the total optical thickness $\tau_{s}$ for a troposphere with an idealized decreasing temperature profile as in Fig. 3 (solid line) and change $\Delta Z_{e}$ of this emission height when the amount of absorbing gas is doubled (dotted line). The dashed line displays the emission height $Z_{e}$ for the isothermal profile. Shades display the function $\tau\left(\tau_{s}, z\right)$, defined as the optical thickness $\tau$ at altitude $z$ when the total optical thickness of the troposphere is $\tau_{s}$. For instance, the emission height $Z_{e}$ almost coincides with the isoline $\tau\left(\tau_{s}, z\right)=1$ for optically thick atmospheres $\left(\tau_{s}>4\right)$.

Starting from the altitude where the pressure is $\left(P_{t}+\right.$ $\left.P_{s}\right) / 2$, the emission height $Z_{e}$ increases when the total optical thickness $\tau_{s}$ increases (Fig. 6). The emission height $Z_{e}$ is commonly approximated as the altitude where the optical thickness is one (Pierrehumbert 2010; Huang and Bani Shahabadi 2014). For the profiles considered here, this approximation is valid as soon as the total optical thickness is larger than about 4 (Fig. 6).

\section{Results with more realistic cloudless atmospheres}

We now abandon previous idealized vertical profiles and consider a realistic cloudless atmosphere, namely the midlatitude summer (MLS) atmospheric profile (McClatchey et al. 1972; Anderson et al. 1986) that has often been used to benchmark radiative codes (Ellingson et al. 1991; Collins et al. 2006; Pincus et al. 2015).

As in the previous section, we consider an atmosphere that ends at the tropopause. The temperature and the pressure at the surface and at the tropopause are close to the previous idealized profile $\left(P_{s}=1013 \mathrm{hPa}, H_{s}=0 \mathrm{~m}\right.$, $T_{s}=294 \mathrm{~K}, P_{t}=190 \mathrm{hPa}, H_{t} \approx 12.6 \mathrm{~km}$, and $T_{t}=$ $218.4 \mathrm{~K})$. The vertical profile of temperature is almost linear with altitude, and therefore the vertical profile of the Planck function at $\nu_{c}=550 \mathrm{~cm}^{-1}$ is not linear with pressure anymore. The volumetric mass density varies according to the perfect gas law and the atmosphere is discretized into 65 vertical layers. The $\mathrm{CO}_{2}$ concentration is 287 ppmv as in Collins et al. (2006). We perform the same computations as in the previous section with this new profile, and the results show few differences relative to those displayed in Figs. 3-6 (not shown). The exact values are slightly modified but all the key features are identical.

\section{a. Single absorption line}

We now consider a narrow frequency range around the center of an absorption line instead of a given frequency. Molecules in gases have discrete energy levels and absorption of photons correspond to transitions between these discrete energy levels. The absorption lines are very numerous (many millions) and not infinitely sharp due to broadening mechanisms. In the Earth troposphere, pressure broadening (also named collision broadening) is the dominant effect and will be the only one considered in a first step. In the vicinity of a line center, the spectral absorption coefficient $k$ varies with frequency according to a Lorentzian profile:

$$
k=\frac{S}{\pi} \frac{\alpha}{\left(\nu-\nu_{c}\right)^{2}+\alpha^{2}}
$$

where $S$ is the line absorption integrated intensity, $\nu$ is the wavenumber, $\nu_{c}$ is the wavenumber of the line center, and $\alpha$ is the half-width at half-height. Lorentz halfwidth is assumed be proportional to $P T^{-0.5}$ :

$$
\alpha(P, T)=\alpha_{0} \frac{P}{P_{0}}\left(\frac{T_{0}}{T}\right)^{0.5},
$$

with $\alpha_{0} \approx 0.1 \mathrm{~cm}^{-1}$ at $P_{0}=1013 \mathrm{hPa}$ and $T_{0}=300 \mathrm{~K}$, which are typical values for the $\mathrm{CO}_{2}$ lines around $550 \mathrm{~cm}^{-1}$. We assume that the line intensity is constant along the vertical and is multiplied by a factor $f=1$ to allow a proportional change in absorption within the whole atmosphere, as in the previous section.

The sensitivity of the spectral radiance at the tropopause to a fractional change in the absorbing gas for a line can be deduced from single frequency results (Fig. 4). A first example is shown for a single and weak absorption line (Fig. 7). The optical thickness at the absorption line center is about 0.75 and decreases rapidly away from the line center. The sensitivity of the radiance is maximum at the line center and is primarily due to the change in absorptivity $\left(\mathscr{F}_{T}^{\prime}\right.$; blue line) as the optical thickness is small.

This picture is very different for a line whose absorption intensity is 12 times larger and that will be referred later as a line of "medium intensity" (Fig. 8). Around the absorption line center, the sensitivity $\tilde{\mathscr{F}}_{\mathcal{T}}^{\prime}$ due to a change in absorptivity is zero as one may expect 

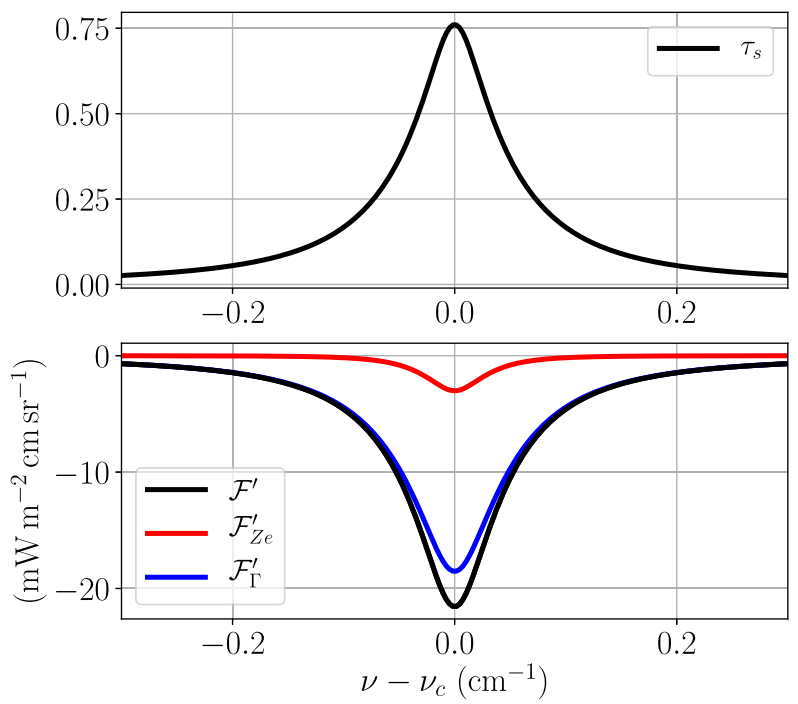

FIG. 7. (top) Total optical thickness $\tau_{s}$ and (bottom) sensitivity of the radiance at the tropopause to a fractional change in absorbing gases as a function of wavenumber $\nu$ for a single weak absorption line and the MLS atmospheric profile. This sensitivity $\left(\mathscr{F}^{\prime}\right.$; black line) is decomposed into the contribution due to the change in emission height $\left(\mathscr{F}_{Z_{e}}^{\prime}\right.$; red line) and due to the change in absorptivity $\left(\mathscr{F}_{\mathcal{T}}^{\prime}\right.$; blue line). The abscissa is the distance from the line center $\left(\nu_{c}=550 \mathrm{~cm}^{-1}\right)$; the line width is $0.1 \mathrm{~cm}^{-1}$ near the surface.

from Fig. 4. In this spectral region the sensitivity $\mathscr{F}_{Z_{e}}^{\prime}$ due to a change in emission height is the dominant factor. Close to the absorption line center, the optical thickness is large and the mean emission height is located close to the tropopause (Fig. 9). An increase of optical thickness has little impact on the emission height. Away from the absorption line center, the sensitivity due to a change in emission height is still the dominant factor and an increase of absorbing gas decreases the radiance at the tropopause and therefore increases the greenhouse effect. Further away from the absorption line center, the sensitivity due to a change in total absorption dominates, and slowly decreases away from the absorption line center.

\section{b. Change in emission height when doubling the amount of absorbing gas}

As a benchmark the amount of absorbing gas is doubled over the whole atmospheric profile. For the idealized atmosphere, the change $\Delta Z_{e}$ in emission height is zero when the optical thickness $\tau_{s}$ of the tropopause is zero, as one may expect (Fig. 6, dotted line). It increases with $\tau_{s}$ up to more than $2 \mathrm{~km}$ for $\tau_{s} \approx 4$ and then decreases with increasing $\tau_{s}$ because the emission height is already close to the tropopause.

For the weak absorption line, the change in emission height is small. It varies from about $20 \mathrm{~m}$ at $0.3 \mathrm{~cm}^{-1}$ from the absorption line center to about $800 \mathrm{~m}$ at the
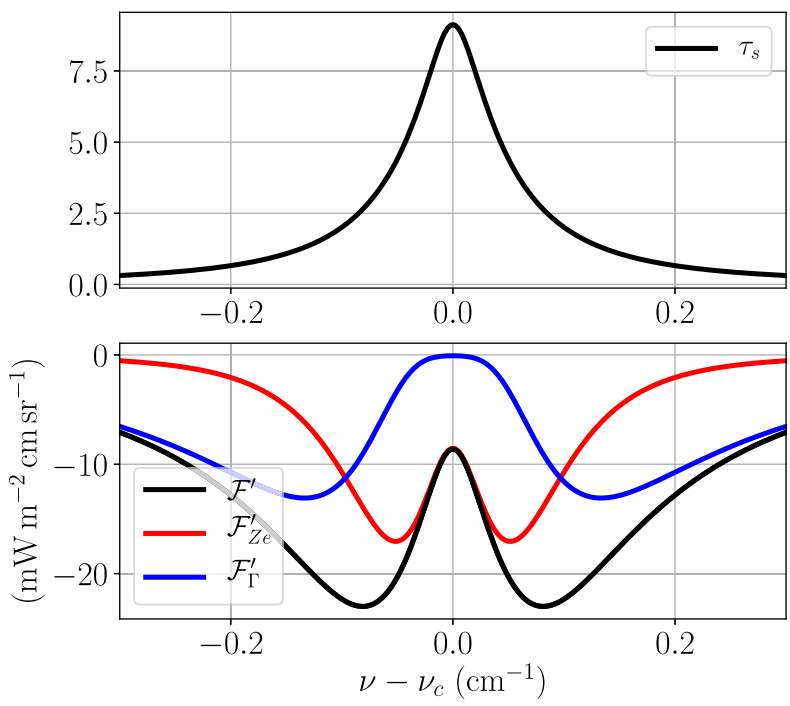

FIG. 8. As in Fig. 7, except that the intensity of the line is larger by a factor of 12 , referred as the line of medium intensity.

absorption line center (not shown). For the absorption line of medium intensity (Fig. 9), the change in emission height increases from about $200 \mathrm{~m}$ at $0.3 \mathrm{~cm}^{-1}$ from the absorption line center up to $2 \mathrm{~km}$ at a wavenumber for which the optical thickness is about 4 and finally decreases to $750 \mathrm{~m}$ at the absorption line center.

From these results, one may expect that for a doubling of the $\mathrm{CO}_{2}$ concentration, the change in emission height

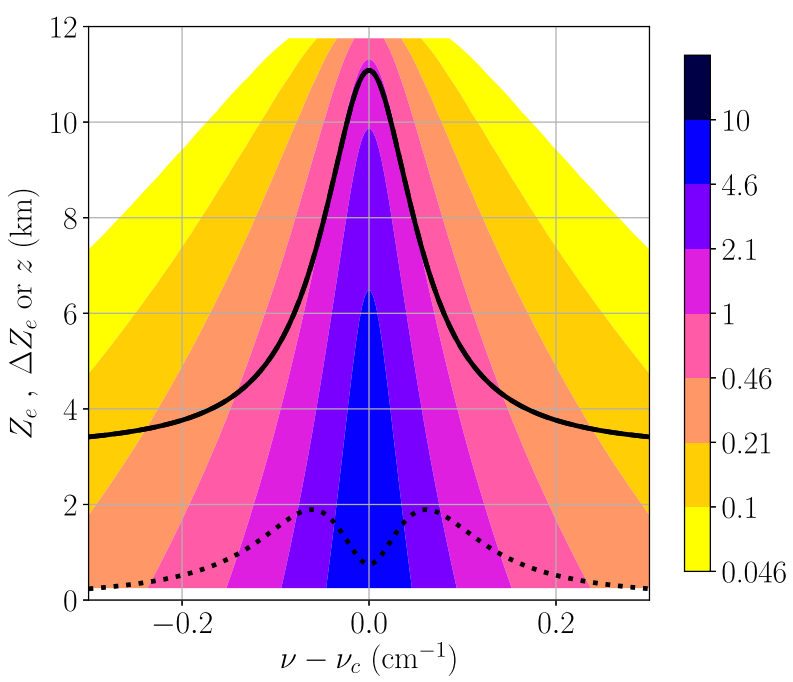

FIG. 9. Emission height $Z_{e}$ (black solid line) as a function of the distance from the absorption line center for the same conditions as in Fig. 8, and change $\Delta Z_{e}$ of this emission height when the amount of absorbing gas is doubled (dotted line). Color shading indicates the optical thickness $\tau$ of the troposphere at altitude $z$ and wavenumber $\nu$. 
will have a value varying from a few tens of meters in spectral regions where the absorptivity is either very weak or very strong to a maximum value of $\approx 1-2 \mathrm{~km}$ in spectral regions where the optical thickness is a few units $\left(\tau_{s} \approx 2-8\right)$.

The results presented until now can be easily reproduced and provide the basis to understand the key phenomena that drives the greenhouse effect. In the next section we will use this understanding to interpret results produced by a comprehensive reference radiation code.

\section{Radiative flux over the whole infrared domain with realistic radiative and thermodynamic properties}

Until now we considered only radiances that allowed us to avoid angular integration. In this section we show how the framework based on radiances can be easily transposed to a framework for irradiance, or radiative flux. We use the classical approximations for pristine atmospheres. The atmosphere is absorbing and nonscattering, perfectly stratified along the horizontal (plane parallel assumption) and the surface has an emissivity of 1 .

\section{a. Framework for radiative flux}

With the above assumptions, the spectral flux $F$ at the tropopause is (Pierrehumbert 2010; Dufresne et al. 2005)

$$
F=\hat{\mathcal{T}}_{s} B_{s}+\int_{P_{t}}^{P_{s}} \frac{\partial \hat{\mathcal{T}}(P)}{\partial P} B(P) d P
$$

where $B=\pi \mathcal{B}$ and $\hat{\mathcal{T}}(P)$ is the spectral hemispherical transmissivity between the pressure level $P$ and the pressure at the tropopause level $P_{t}$ :

$$
\hat{\mathcal{T}}(P)=2 \int_{0}^{1} \exp [-\tau(P, \mu)] \mu d \mu .
$$

Here, $\tau(P, \mu)$ is the spectral directional optical thickness between the tropopause and the pressure level $P$ :

$$
\tau(P, \mu)=\left|\int_{P_{t}}^{P} \frac{f k(P)}{g \mu} d P\right|,
$$

where $\mu$ is the cosine of the zenith angle, $k(P)$ the specific absorption coefficient at level of pressure $P$, and $f=1$ is a multiplicative factor as in the previous sections. Equation (17) is similar to Eq. (6) previously used, except that the radiances $(\mathscr{F}, \mathcal{B})$ have been replaced by the irradiance (or flux) $(F, B)$, and the directional transmissivity $\mathcal{T}$ has been replaced by the hemispherical transmissivity $\hat{\mathcal{T}}$. With these replacements, one can show that Eqs. (8)-(14) can be directly adapted to fluxes. For instance, Eq. (17) can be rewritten as

$$
\begin{aligned}
F & =\hat{\mathcal{T}}_{s} B_{s}+\left(1-\hat{\mathcal{T}}_{s}\right) B_{e}, \\
B_{e} & =\int_{P_{t}}^{P_{s}} B(P) \omega(P) d P, \quad \text { and } \\
\omega(P) & =\frac{1}{1-\hat{\mathcal{T}}_{s}} \frac{\partial \hat{\mathcal{T}}(P)}{\partial P},
\end{aligned}
$$

which can be compared with Eqs. (8a)-(8c). In Eq. (20a), $\hat{\mathcal{T}}_{s} B_{s}$ is termed the surface transmitted irradiance in Costa and Shine (2012). Applying the same replacements to Eqs. (10a)-(10c) allows us to split the sensitivity of the flux at the tropopause $F^{\prime}$ into a contribution $F_{\hat{\mathcal{T}}}^{\prime}$ due to the change in absorptivity and a contribution $F_{Z_{e}}^{\prime}$ due to the change in emission height.

Many radiative codes do not compute the sensitivity $F^{\prime}$ directly, and a difference in radiances can therefore be more suitable. For two atmospheres $i=1,2$ that only differ by the amount of absorbing gases, the flux at the tropopause reads

$$
F_{i}=\hat{\mathcal{T}}_{s, i} B_{s}+\left(1-\hat{\mathcal{T}}_{s, i}\right) B_{e, i}
$$

One can show (see appendix B) that the difference between the two fluxes $\Delta F=F_{2}-F_{1}$ reads as

$$
\begin{aligned}
\Delta F & =\Delta F_{\hat{\mathcal{T}}}+\Delta F_{Z_{e}}, \\
\Delta F_{\hat{\mathcal{T}}} & =\left(\hat{\mathcal{T}}_{s, 2}-\hat{\mathcal{T}}_{s, 1}\right)\left(B_{s}-B_{e, 1}\right), \quad \text { and } \\
\Delta F_{Z_{e}} & =\left(1-\hat{\mathcal{T}}_{s, 2}\right)\left(B_{e, 2}-B_{e, 1}\right) .
\end{aligned}
$$

Thus, $\Delta F_{\hat{\mathcal{T}}}$ quantifies the effect of the change in absorptivity and $\Delta F_{Z_{e}}$ quantifies the effect of the change in emission height. If $F, B_{s}$, and $\hat{\mathcal{T}}_{s}$ are known, $B_{e, 2}$ and $B_{e, 1}$ can be computed using Eq. (21), and therefore Eqs. (22b) and (22c) can be used to compute $\Delta F_{\hat{\mathcal{T}}}$ and $\Delta F_{Z_{e}}$. Therefore, any radiative code, no matter how complex, that computes $F, B_{s}$, and $\hat{\mathcal{T}}_{s}$, which is generally the case, can be used to compute the changes $\Delta F_{\hat{\mathcal{T}}}$ of the flux at the tropopause that is due to the change in absorptivity and the change $\Delta F_{Z_{e}}$ that is due to the change in emission height. However, the change in emission height itself is more difficult to compute as it requires the use of Eq. (14), which is not straightforward for many radiative codes. This is one of the reasons why we used a radiative code based on the net exchange formulation (NEF) (Green 1967; Cherkaoui et al. 1996; Dufresne et al. 2005). 
TABLE 1. Difference of the net flux $\left(\mathrm{W} \mathrm{m}^{-2}\right)$ at the TOA, at $200 \mathrm{hPa}[\Delta \bar{F}(200)]$, and at the surface $\left(\Delta \bar{F}_{s}\right)$ for a $\mathrm{CO}_{2}$ doubling and for an increase of $\mathrm{H}_{2} \mathrm{O}$ by $20 \%$ for the MLS atmospheric profile and for the thermal infrared spectral interval $\left(100-2500 \mathrm{~cm}^{-1}\right.$; i.e., $\left.4-100 \mu \mathrm{m}\right)$. The results computed with our model (label KS) are compared with those published by Collins et al. (2006) (label C06) for an ensemble of line-by-line models using the same atmospheric profiles (see section 4b).

\begin{tabular}{|c|c|c|c|c|c|c|}
\hline \multirow[b]{2}{*}{ Expts } & \multicolumn{2}{|c|}{$\Delta \bar{F}$ TOA } & \multicolumn{2}{|c|}{$\Delta \bar{F}(200)$} & \multicolumn{2}{|c|}{$\Delta \bar{F}_{s}$} \\
\hline & KS & $\mathrm{C} 06$ & KS & $\mathrm{C} 06$ & $\mathrm{KS}$ & $\mathrm{C} 06$ \\
\hline $2 \times \mathrm{CO}_{2}(2 \mathrm{~b}-1 \mathrm{a})$ & 2.81 & $2.80 \pm 0.06$ & 5.57 & $5.48 \pm 0.07$ & 1.67 & $1.64 \pm 0.04$ \\
\hline $1.2 \times \mathrm{H}_{2} \mathrm{O}(4 \mathrm{a}-2 \mathrm{~b})$ & 3.71 & $3.78 \pm 0.1$ & 4.60 & $4.57 \pm 0.14$ & 11.43 & $11.52 \pm 0.40$ \\
\hline
\end{tabular}

\section{b. A reference line-by-line model based on a net exchange formulation}

The line-by-line radiative model we use is presented in Eymet et al. (2016) and its main originality is to rely on the NEF. In a first step (Kspectrum code), a synthetic high-resolution (typically $0.0005 \mathrm{~cm}^{-1}$ ) absorption spectrum is computed for the required atmospheric profile using the HITRAN 2012 molecular spectroscopic database (Rothman et al. 2013) with Voigt line profiles. For $\mathrm{CO}_{2}$, sub-Lorentzian corrections are taken into account. For $\mathrm{H}_{2} \mathrm{O}$, the CKD continuum is used with a $25-\mathrm{cm}^{-1}$ truncation and removing the "base" of each transition (Clough et al. 1989; Mlawer et al. 2012). In a second step (HR_PPart code), radiative transfer is computed based on 1D (over a single line of sight) or 3D (angularly integrated) analytical expressions of spectral radiative net exchange rates and spectral radiative fluxes. We compute the radiative forcing for $\mathrm{CO}_{2}$ and $\mathrm{H}_{2} \mathrm{O}$ changes on the basis of the experiments defined in Collins et al. (2006): the reference experiment, which is the MLS atmospheric profile with a $\mathrm{CO}_{2}$ concentration of 287 ppmv (experiment 1a), an experiment in which the $\mathrm{CO}_{2}$ concentration is doubled (experiment $2 \mathrm{~b}$ ), and an experiment in which the $\mathrm{CO}_{2}$ concentration is doubled and the concentration of $\mathrm{H}_{2} \mathrm{O}$ is increased by $20 \%$ (experiment $4 \mathrm{a}$ ). In this example, the only absorbing gases considered are $\mathrm{H}_{2} \mathrm{O}$, $\mathrm{CO}_{2}$, and ozone, and the troposphere is discretized into 31 vertical layers. The results compare well to those published by Collins et al. (2006), as shown in Table 1 .

\section{c. Results for a realistic atmospheric profile}

We use the same MLS atmospheric profile and consider only the troposphere, from the surface $\left(P_{s}=\right.$ $1013 \mathrm{hPa}, H_{s}=0 \mathrm{~m}$, and $\left.T_{s}=294 \mathrm{~K}\right)$ to the tropopause $\left(P_{t}=190 \mathrm{hPa}, H_{t} \approx 12.6 \mathrm{~km}\right.$, and $\left.T_{t}=218.4 \mathrm{~K}\right)$, as presented above.

We first focus on two $\mathrm{CO}_{2}$ weak absorbing lines. In Fig. 10, and only in this figure, we exclude absorption by the $\mathrm{H}_{2} \mathrm{O}$ continuum in order to have an optical thickness that is as small as possible. For the weaker absorption line for which the optical thickness is always less than one (Fig. 10, left column), the shape of the optical thickness resembles that of the idealized one (Fig. 7). The optical thickness is low $\left(\tau_{s} \ll 1\right)$ and the emission height is about $2-3 \mathrm{~km}$, as expected from Fig. 9. When doubling the $\mathrm{CO}_{2}$ concentration, the change in optical thickness is almost equal to the value for the reference atmosphere, the difference is due to some absorption by $\mathrm{H}_{2} \mathrm{O}$. The change in emission height is less than $100 \mathrm{~m}$ at wavenumbers far away from the absorption line center and increases to a few hundred meters at the absorption line center. The change in the tropopause irradiance is largely dominated by the contribution of the change in absorptivity.

For a more absorbing line with a companion weak absorbing line (Fig. 10, right column), the emission height is about $2-3 \mathrm{~km}$ far from the absorption line center, where the optical thickness is below 1 . At the absorption line center, the emission height reaches $8 \mathrm{~km}$, which is closer to the tropopause. When doubling the $\mathrm{CO}_{2}$ concentration, the change in emission height is a few hundred meters far from the absorption line center to more than a kilometer at the absorption line center. The change in the tropopause irradiance is dominated by the contribution of the change in emission height.

The results we obtained with the various idealized configurations are consistent with those we obtained with the reference model. The understanding we gained with the idealized examples can be applied to interpret the results with much more complex and realistic models.

We now consider the "thermal infrared" spectral interval from 100 to $2500 \mathrm{~cm}^{-1}(4-100 \mu \mathrm{m})$. In Figs. 11 and 12 (and later in Fig. 14), variables are smoothed on a 10$\mathrm{cm}^{-1}$ spectral interval to make the figure more readable. The spectral dependencies of the radiative flux at the TOA, at the tropopause, within the atmosphere, and of the radiative cooling rate in the atmosphere, as well as how they change when changing the $\mathrm{CO}_{2}$ concentration, have already been addressed in many studies (Kiehl and Ramanathan 1983; Kiehl 1983; Charlock 1984; Clough and Iacono 1995; Harries et al. 2001; Huang 2013). Mlynczak et al. (2016) show that these results were remarkably insensitive to known uncertainties in the main $\mathrm{CO}_{2}$ spectroscopic parameters. Zhong and Haigh (2013) showed how the flux at the TOA varies over a wide 

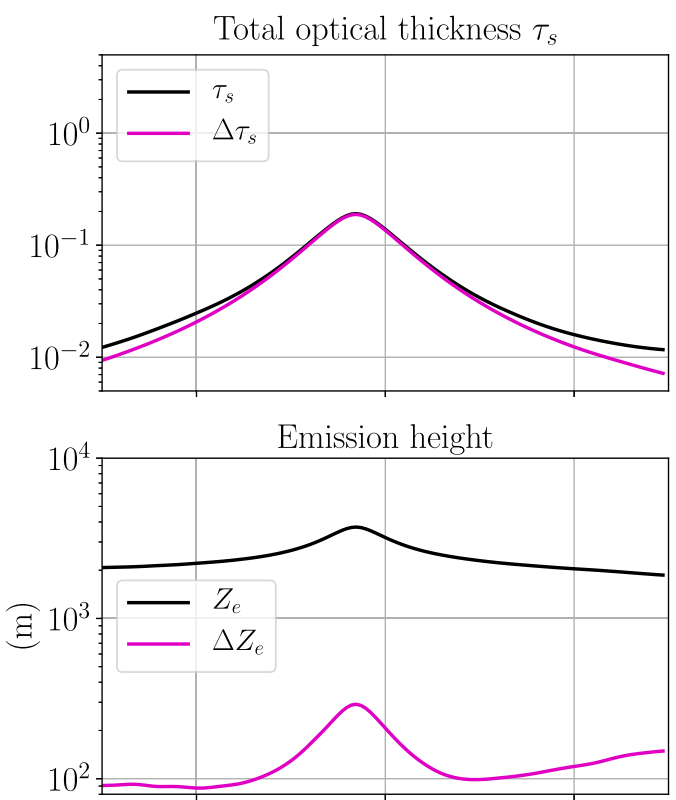

Sensitivity of outgoing flux

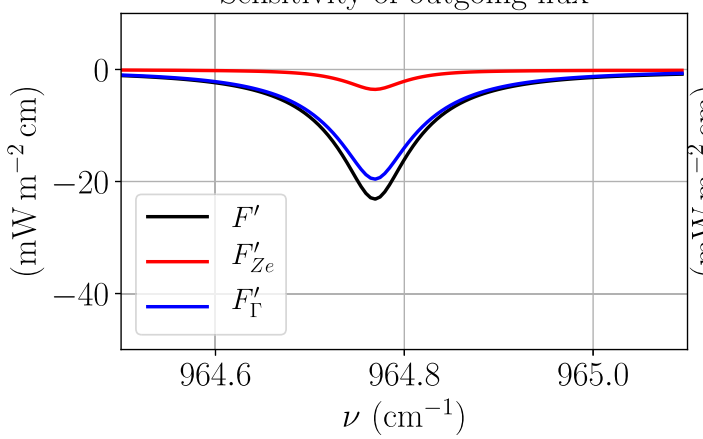

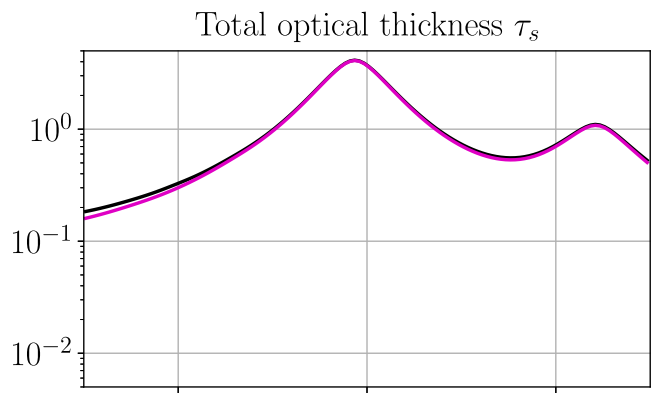

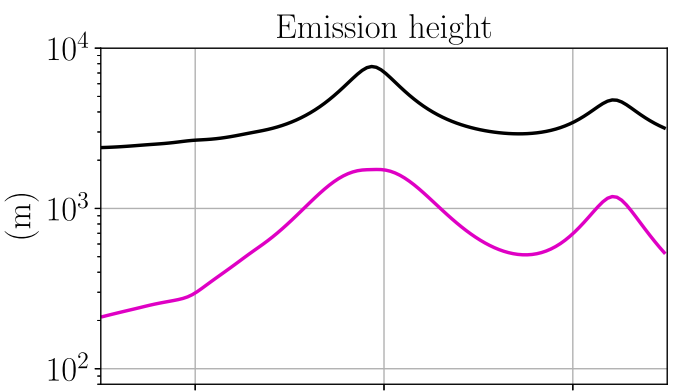

Sensitivity of outgoing flux

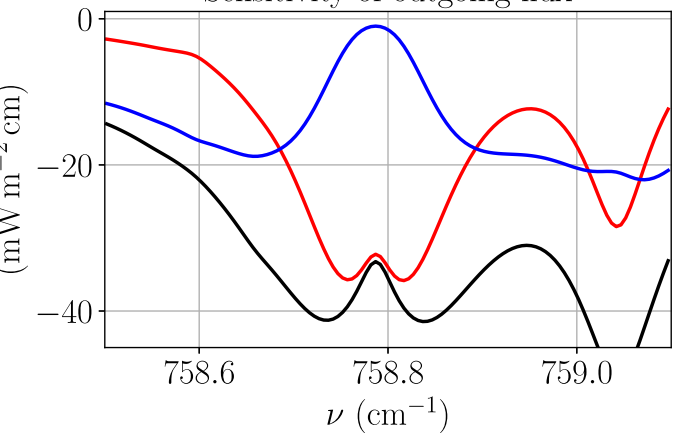

FIG. 10. (top) Total optical thickness $\tau_{s}$ of the troposphere (black line) and its change $\Delta \tau_{s}$ (magenta line) for a $\mathrm{CO}_{2}$ doubling, (middle) emission height $Z_{e}$ (black line) and its change $\Delta Z_{e}$ (magenta line) for a $\mathrm{CO}_{2}$ doubling, and (bottom) sensitivity $F^{\prime}$ of the flux at the tropopause (black line) to a fractional change in $\mathrm{CO}_{2}$ and contributions of change in absorptivity (blue line: $F_{\hat{\mathcal{T}}}^{\prime}$ ) and in emission height (red line: $F_{Z_{e}}^{\prime}$ ) as a function of wavenumber $\left(\mathrm{cm}^{-1}\right)$. Shown are (left) a weak absorption line $\left(964.5-965.1 \mathrm{~cm}^{-1}\right)$ and (right) an intermediate absorption line with a companion weak absorbing line $\left(758.5-759.1 \mathrm{~cm}^{-1}\right)$.

range of $\mathrm{CO}_{2}$ values, and they showed that the spectral response is very different depending on the $\mathrm{CO}_{2}$ concentration. Here we consider the response for an atmosphere with a $\mathrm{CO}_{2}$ concentration close to its preindustrial value (287 ppmv).

The total optical thickness $\tau_{s}$ (Fig. 11a, black line) is primarily due to $\mathrm{H}_{2} \mathrm{O}$ absorption, except around 660 and $2300 \mathrm{~cm}^{-1}$ where the two $\mathrm{CO}_{2}$ strong absorption band systems at 15 and $4.3 \mu \mathrm{m}$ (magenta) are dominant. The total optical thickness varies over many orders of magnitude, from about 1 in the atmospheric window (between 800 and $1200 \mathrm{~cm}^{-1}$ ) to $10^{4}-10^{5}$ in the $\mathrm{H}_{2} \mathrm{O}$ and $\mathrm{CO}_{2}$ absorption bands. When the data are not smoothed, the range is even larger, from a few tenths up to $10^{6}$.
The emission height (Fig. 11b) almost increases with the logarithm of the total optical thickness $\tau_{s}$ (Huang and Bani Shahabadi 2014). It varies for $2 \mathrm{~km}$ in the atmospheric window up to $12 \mathrm{~km}$ (i.e., almost the tropopause height) in the spectral region where the optical thickness is very high, especially for the $\mathrm{CO}_{2}$ bands. For the same optical thickness, the emission height in the $\mathrm{CO}_{2}$ absorption bands are larger than for the $\mathrm{H}_{2} \mathrm{O}$ absorption bands as the $\mathrm{CO}_{2}$ concentration is uniform over the whole troposphere whereas the $\mathrm{H}_{2} \mathrm{O}$ concentration strongly decreases with height.

We define the emission temperature as the temperature for which the Planck function is equal to $B_{e}$ defined by Eq. (20b). The emission temperature (Fig. 11c) directly follows the evolution of the emission height. The 


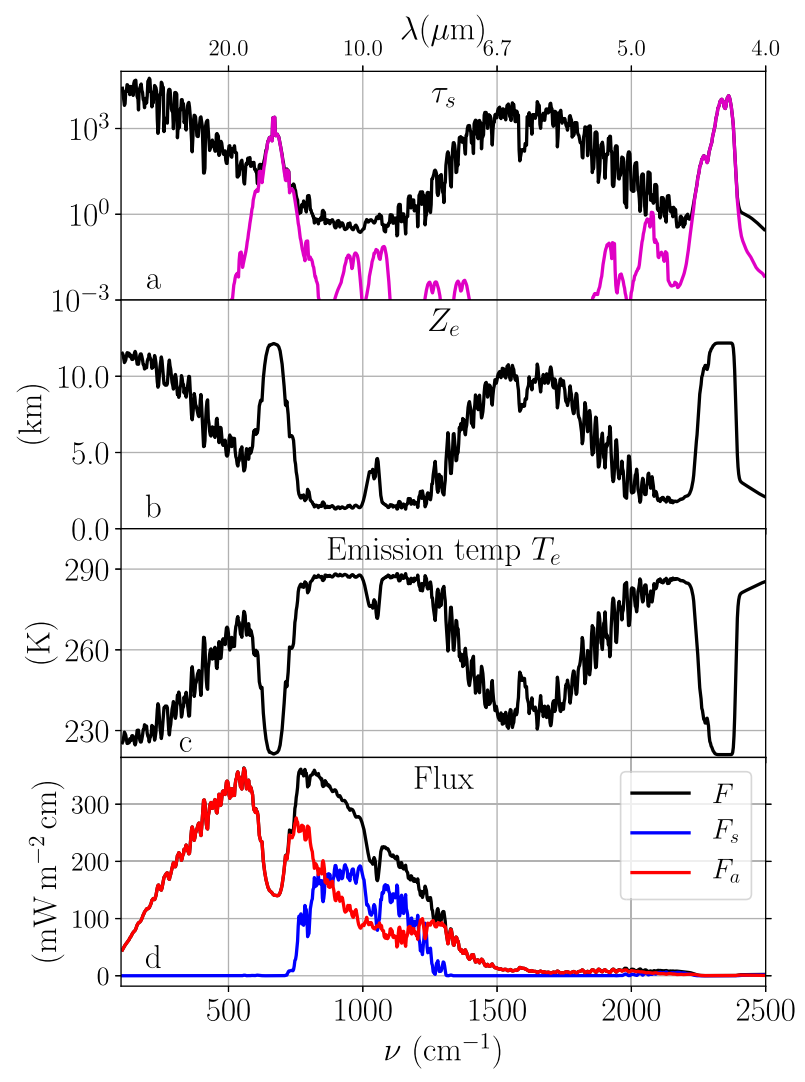

FIG. 11. (a) Optical thickness $\tau_{s}$ (black line: total optical thickness; magenta line: optical thickness due to $\mathrm{CO}_{2}$ ), (b) emission height $Z_{e}$, (c) emission temperature $T_{e}$, and (d) upward radiative flux at the tropopause (black: total, $\mathscr{F}$; blue: emitted by the surface, $\mathscr{F}_{s}$; red: emitted by the troposphere, $\mathscr{F}_{a}$ ) for the MLS atmospheric profile. The abscissa is given in wavenumber $\left(\mathrm{cm}^{-1}\right)$ at the bottom and in wavelength $(\mu \mathrm{m})$ at the top. Variables are smoothed on a $10 \mathrm{~cm}^{-1}$ spectral interval.

dependence is about $7 \mathrm{~K} \mathrm{~km}^{-1}$, as one may expect from the value of the temperature gradient in the troposphere. The upward flux at the tropopause may have been emitted either from the surface or from the troposphere [Eqs. (17) and (6)]. One can see in Fig. 11d that almost all the flux at the tropopause has been emitted by the troposphere, except in the atmospheric window where the emissions by the surface and the troposphere contribute almost equally (Costa and Shine 2012).

The ozone absorption band around $1050 \mathrm{~cm}^{-1}$ has a specific signature as ozone is mainly located in the higher part of the troposphere. This band has little impact on the optical thickness but has a visible signature on the emission height, the emission temperature, and the outgoing flux (Fig. 11).

Figure 12 displays the changes in total optical thickness, emission height, emission temperature, and upward flux at the tropopause when doubling the $\mathrm{CO}_{2}$ concentration or when increasing the $\mathrm{H}_{2} \mathrm{O}$ concentration by

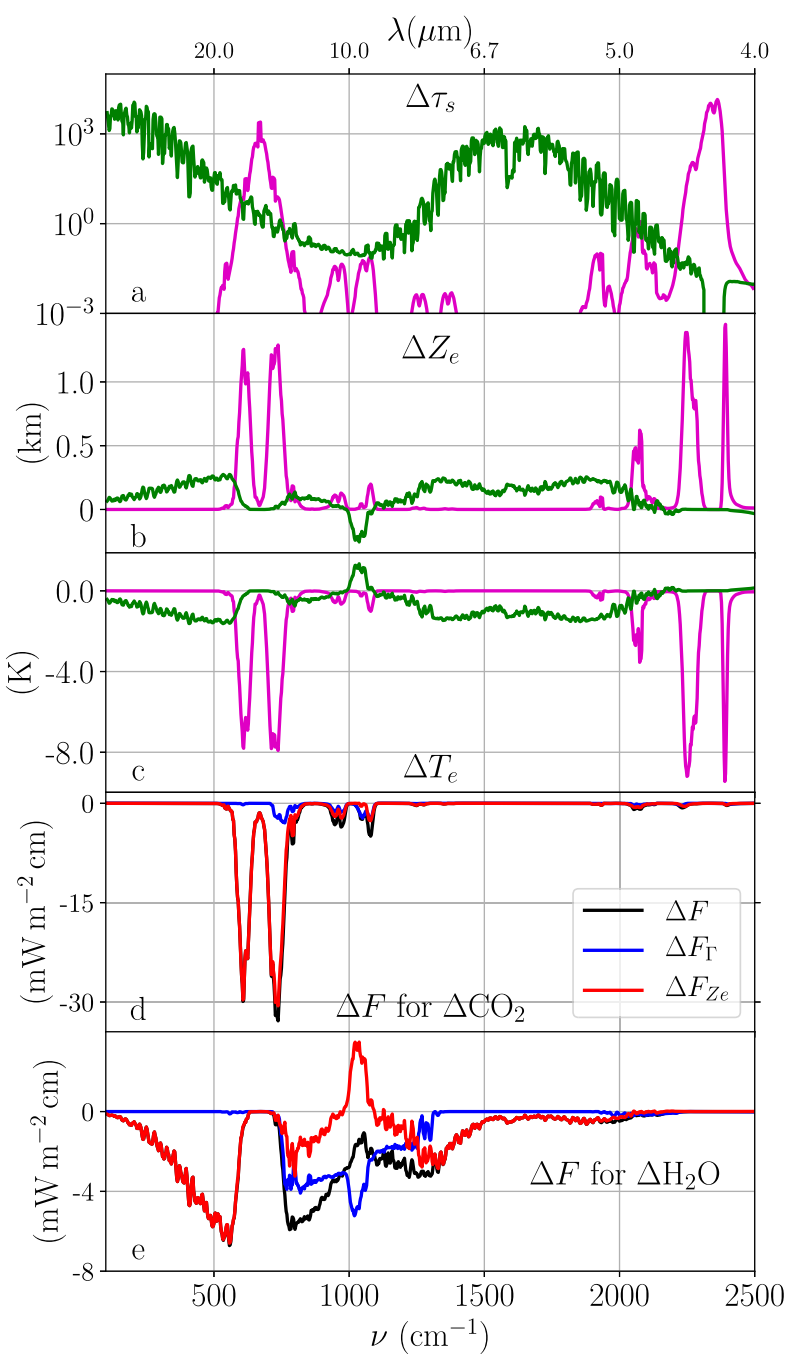

FIG. 12. Changes due to a $\mathrm{CO}_{2}$ doubling (magenta line) and to an increase by $20 \%$ of the $\mathrm{H}_{2} \mathrm{O}$ concentration (green line) of the (a) optical thickness $\tau_{s}$, (b) emission height $Z_{e}$, and (c) emission temperature $T_{e}$ for the same atmospheric profile as in Fig. 11. The changes $\Delta F$ of the flux at the tropopause (black line) and the contributions of the change in atmospheric absorptivity (blue line, $\Delta F_{\hat{T}}$ ) and in emission height (red line; $\Delta F_{Z_{e}}$ ) are shown for (d) $\mathrm{CO}_{2}$ and (e) $\mathrm{H}_{2} \mathrm{O}$. The abscissa is given in wavenumber $\left(\mathrm{cm}^{-1}\right)$ at the bottom and in wavelength $(\mu \mathrm{m})$ at the top. Variables are smoothed on a $10-\mathrm{cm}^{-1}$ spectral interval.

$20 \%$. For the $\mathrm{CO}_{2} 15-\mu \mathrm{m}$ band system $\left(660 \mathrm{~cm}^{-1}\right)$, the change in emission height, emission temperature, and tropopause flux is maximum on the edges of the band (Figs. 12b-d), where the $\mathrm{CO}_{2}$ optical thickness is about a few units (Fig. 11a). In these spectral regions, the change in emission height is about $1 \mathrm{~km}$ and the change in emission temperature is about $7 \mathrm{~K}$. The change in emission height is almost zero at the band center as the emission height is already close to the tropopause (i.e., close to the maximum height). The change in the flux at 
the tropopause is almost only due to the change in emission height (Fig. 12d). For the $4.3-\mu \mathrm{m}\left(2300 \mathrm{~cm}^{-1}\right)$ $\mathrm{CO}_{2}$ band, the changes in emission height and emission temperature resemble those for the $15-\mu \mathrm{m}\left(660 \mathrm{~cm}^{-1}\right)$ band, but these changes have almost no impact on the tropopause flux as the Planck function is almost zero at these wavenumbers for the atmospheric temperature. In addition to these two very strong absorption bands, $\mathrm{CO}_{2}$ also has some minor bands that produce small changes in emission height, emission temperature, and tropopause flux. In the spectral domain of these minor bands, the optical thickness is small (about $10^{-1}$ ) and is due to absorption by both $\mathrm{H}_{2} \mathrm{O}$ and $\mathrm{CO}_{2}$. As a result, both the change in emission height and in absorptivity play a comparable role, whereas the change in absorptivity would have had a dominant role if $\mathrm{CO}_{2}$ were the only absorbing gas. Note that this holds for the current atmosphere but not for an atmosphere with very high $\mathrm{CO}_{2}$ concentration: these "minor" bands contribute to the $\mathrm{CO}_{2}$ forcing by about $6 \%$ in current conditions, but they contribute by about $25 \%$ for $\mathrm{CO}_{2}$ concentration that are 100 times larger (Augustsson and Ramanathan 1977; Zhong and Haigh 2013).

As the change $\Delta Z_{e}$ in emission height strongly varies with wavenumber, we define its average value in two ways. The first is the broadband average $\left\langle\Delta Z_{e}\right\rangle_{P}$, where $\Delta Z_{e}$ is weighted by the Planck function at surface temperature, as for the broadband absorptivity shown on Fig. 1a. We found a value of $150 \mathrm{~m}$, exactly as Held and Soden (2000). As explained in this article, the broadband change in emission height can be directly used to compute the radiative forcing. However, the change in the flux at the tropopause is different from zero only in limited spectral regions where $\Delta Z_{e}$ is also large (Fig. 12). Therefore we define a second average, $\left\langle\Delta Z_{e}\right\rangle_{F}$-namely, the "forcing average" change in emission height defined as the average of $\Delta Z_{e}$ weighted by $\Delta F_{Z_{e}}$ :

$$
\left\langle\Delta Z_{e}\right\rangle_{F}=\frac{\int_{0}^{\infty} \Delta Z_{e}(\nu) \Delta F_{Z_{e}}(\nu) d \nu}{\int_{0}^{\infty} \Delta F_{Z_{e}}(\nu) d \nu} .
$$

This quantity is the change in emission height that actually contributes to the radiative forcing. We obtain a value of $1025 \mathrm{~m}$, which is much larger than the broadband mean. The change in $\mathrm{CO}_{2}$ concentration impacts the flux at the tropopause in the very few spectral regions where the optical thickness of the atmosphere is about a few units. In these spectral regions the change in emission height is on average $1025 \mathrm{~m}$. The mean emission height itself is less sensitive to the average method: The broadband emission height is $5800 \mathrm{~m}$ whereas the "forcing average" emission height is $6100 \mathrm{~m}$.

When the $\mathrm{H}_{2} \mathrm{O}$ concentration is increased, the change in emission height is about $200 \mathrm{~m}$ (Fig. 12b) over spectral intervals that are much wider $\left(100-600\right.$ and $\left.1300-2000 \mathrm{~cm}^{-1}\right)$ than for $\mathrm{CO}_{2}$. In these intervals the absorption by $\mathrm{H}_{2} \mathrm{O}$ is strong and the change of the flux at the tropopause is almost only due to the change in emission height (Fig. 12e). In spectral regions where absorption by $\mathrm{CO}_{2}$ dominates $\left(600-750 \mathrm{~cm}^{-1}\right)$, the change in $\mathrm{H}_{2} \mathrm{O}$ is completely masked by the $\mathrm{CO}_{2}$ absorption. In most of the atmospheric window $\left(750-1300 \mathrm{~cm}^{-1}\right)$, the change in emission height is small $(<100 \mathrm{~m})$ and the change of the flux at the tropopause is mainly due to the change in absorptivity, with a significant contribution of the water vapor continuum (Costa and Shine 2012). An exception is around $1050 \mathrm{~cm}^{-1}$ where ozone absorbs. In this spectral region both the ozone and the water vapor emit radiation and the emission height includes both the contribution of ozone, which is mainly located in the high troposphere, and the contribution of water vapor, which is mainly located in the lower troposphere. When the $\mathrm{H}_{2} \mathrm{O}$ concentration increases, the radiation emitted by $\mathrm{H}_{2} \mathrm{O}$ that reaches the tropopause increases whereas the radiation emitted by ozone that reaches the tropopause does not change. As a result the emission height decreases by about $200 \mathrm{~m}$ (Fig. 12b), the emission temperature increases (Fig. 12c), and the contribution of the change in emission height to the flux at the tropopause is positive (Fig. 12e).

When considering the radiative flux over the whole thermal infrared domain, the decrease of the flux at the tropopause due to an increase of $\mathrm{CO}_{2}$ is primarily due (by about $90 \%$, Table 2) to the change in emission height, the change in absorptivity playing a minor role (about $10 \%$ ). For an increase of water vapor, the change in absorptivity plays a more important role (about $40 \%$ ) but the change in emission height still plays the dominant role $(\approx 60 \%)$. However, this significant contribution of the change in absorptivity for $\mathrm{H}_{2} \mathrm{O}$ is primarily due to the $\mathrm{H}_{2} \mathrm{O}$ continuum. When the continuum is suppressed, the change in emission height is as high as $80 \%$ and the contribution of the change in absorptivity reduces to $20 \%$.

\section{$d$. Including the stratosphere}

So far and for simplicity we considered an atmosphere that extends from the surface to the tropopause, and therefore in which the vertical temperature gradient is always negative and driven by the convective adjustment. We now consider an atmosphere that extends to an altitude of $100 \mathrm{~km}$ and will show that the main results are still valid when the temperature adjustment of the stratosphere is taken into account. 
TABLE 2. Difference $\Delta \bar{F}$ of the upward flux at the tropopause, difference $\Delta \bar{F}_{Z_{e}}$ of this flux due to change in emission height, difference $\Delta \bar{F}_{\hat{\mathcal{T}}}$ due to change in absorptivity, relative contribution of each of the changes $\left(\Delta \bar{F}_{Z_{e}}\right.$ and $\left.\Delta \bar{F}_{\hat{\mathcal{T}}}\right)$ to the total $\Delta \bar{F}$, and change $\Delta \hat{\mathcal{T}}_{s}$ of the broadband transmissivity of the atmosphere. The differences are computed for a $\mathrm{CO}_{2}$ doubling $\left(2 \mathrm{~b}-1 \mathrm{a}\right.$; first row) and an increase of $\mathrm{H}_{2} \mathrm{O}$ by $20 \%(4 a-2 b$; second row) for the atmospheric profiles (Atm profile) presented in the text (section $4 b$ ).

\begin{tabular}{|c|c|c|c|c|c|c|c|}
\hline Atm profile & Expts & $\begin{array}{c}\Delta \bar{F} \\
\left(\mathrm{~W} \mathrm{~m}^{-2}\right)\end{array}$ & $\begin{array}{c}\Delta \bar{F}_{Z_{e}} \\
\left(\mathrm{~W} \mathrm{~m}^{-2}\right)\end{array}$ & $\begin{array}{c}\Delta \bar{F}_{\hat{\mathcal{T}}} \\
\left(\mathrm{W} \mathrm{m}^{-2}\right)\end{array}$ & $\begin{array}{l}\Delta \bar{F}_{Z_{e}} / \Delta \bar{F} \\
\quad(-)\end{array}$ & $\begin{array}{l}\Delta \bar{F}_{\hat{\mathcal{T}}} / \Delta \bar{F} \\
\quad(-)\end{array}$ & $\begin{array}{l}\Delta \overline{\hat{\mathcal{T}}}_{s} \\
(-)\end{array}$ \\
\hline MLS & $\begin{array}{l}2 \times \mathrm{CO}_{2}(2 \mathrm{~b}-1 \mathrm{a}) \\
1.2 \times \mathrm{H}_{2} \mathrm{O}(4 \mathrm{a}-2 \mathrm{~b})\end{array}$ & $\begin{array}{l}-3.83 \\
-3.78\end{array}$ & $\begin{array}{l}-3.46 \\
-2.21\end{array}$ & $\begin{array}{l}-0.37 \\
-1.57\end{array}$ & $\begin{array}{l}0.90 \\
0.58\end{array}$ & $\begin{array}{l}0.10 \\
0.42\end{array}$ & $\begin{array}{l}-4.110^{-3} \\
-2.710^{-2}\end{array}$ \\
\hline
\end{tabular}

In the stratosphere, the radiative cooling is compensated by the dynamic heating with a relaxation time of a few months. As the dynamics in the troposphere and in the stratosphere are weakly coupled, it has been shown (Hansen et al. 1981, 1997; Stuber et al. 2001; Forster et al. 2007) that it is more relevant to compute the radiative forcing after allowing stratospheric temperatures to adjust to a new radiative equilibrium than to compute the radiative forcing with a fixed stratospheric temperature. The stratospheric temperature adjustment is computed assuming no change in stratospheric dynamics as follows: after computing the radiative budget $S_{1}(z)$ at each altitude $z$ for the reference concentration and temperature, the radiative budget $S_{2}(z)$ at each altitude $z$ is computed with the same temperature profile but a modified $\mathrm{CO}_{2}$ concentration. The temperature in the stratosphere is then adjusted until $S_{2}(z) \approx S_{1}(z)$ at each altitude $z$ of the stratosphere. The results we obtain for the MLS profile and a doubling of the $\mathrm{CO}_{2}$ concentration are shown in Fig. 13. By construction the temperature in the troposphere does not change. The temperature in the stratosphere decreases as expected (Hansen et al. 1997; Stuber et al. 2001), with a temperature cooling of $5-10 \mathrm{~K}$.

Relative to the troposphere-only case (Fig. 11), including the stratosphere increases the emission height in the center of the $\mathrm{CO}_{2}$ absorption band systems at 15 and $4.3 \mu \mathrm{m}$ where the emission height reaches values up to 30 to $40 \mathrm{~km}$ (Fig. 14a). This height is even larger when looking at the full resolution data (not shown). Including the stratosphere increases the optical thickness around the $9.7 \mu \mathrm{m}\left(\approx 1050 \mathrm{~cm}^{-1}\right) \mathrm{O}_{3}$ absorption band by a few units, which has a significant impact on both the emission height and the flux at the TOA (Figs. 14a,b).

When doubling the $\mathrm{CO}_{2}$ concentration, the change in emission height (Fig. 14c) is comparable to the case without stratosphere (Fig. 12b) except in the 15- and 4.3- $\mu \mathrm{m} \mathrm{CO}_{2}$ absorption bands. At these band centers, the emission height can now be larger than the tropopause height; the increase in emission height is not blocked anymore and it has an almost constant value of about $3 \mathrm{~km}$. One can show that the change in emission height is almost constant for a well-mixed absorption gas when absorption is saturated because the emission height is then close to the height where the optical thickness is equal to one (Fig. 6). This large change in emission height has a clear signature on the change of the flux at the TOA for the $15-\mu \mathrm{m} \mathrm{CO}_{2}$ absorption band. At the absorption band center, a higher emission height leads to an increase of the outgoing flux because the temperature vertical gradient in the stratosphere is positive. However, this happens only if the temperature in the stratosphere is fixed (Fig. 14d, dashed line) as already shown (Kiehl 1983; Charlock 1984). If the temperature of the stratosphere is adjusted as explained above, the decrease of temperature in the stratosphere leads to a decrease of the emitted radiation. As a result, the change in the outgoing flux at the center of the $15-\mu \mathrm{m} \mathrm{CO}_{2}$ absorption band is slightly negative. The pattern of the change of the spectral flux around the $15-\mu \mathrm{m} \mathrm{CO}_{2}$ absorption band is similar if the atmosphere only extends up to the tropopause (Fig. 12d) and if the atmosphere extends

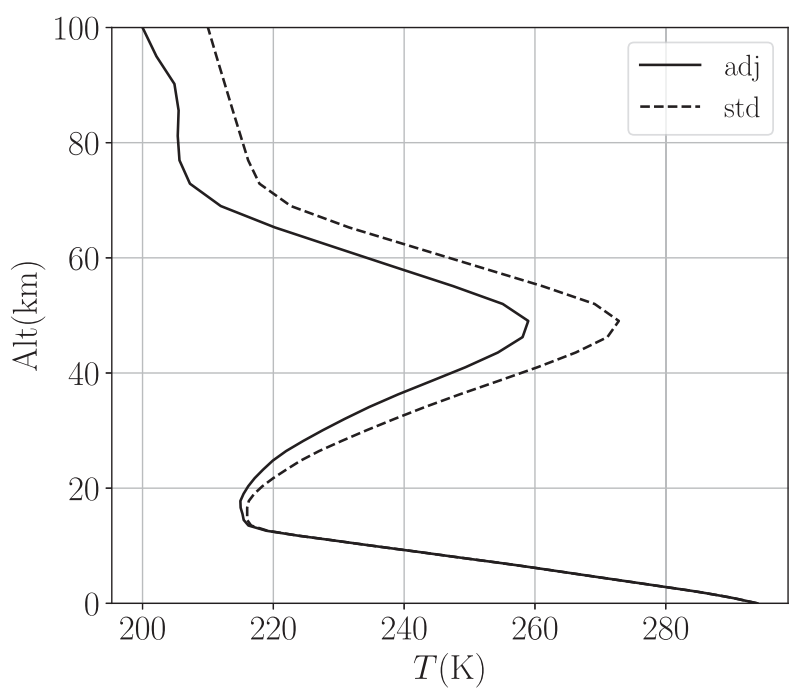

FIG. 13. Temperature as a function of altitude for the full MLS atmospheric profile, i.e., including the stratosphere. Both the reference temperature (dashed line) and the temperature after the stratosphere has adjusted to a doubling of the $\mathrm{CO}_{2}$ concentration (solid line) are shown. 


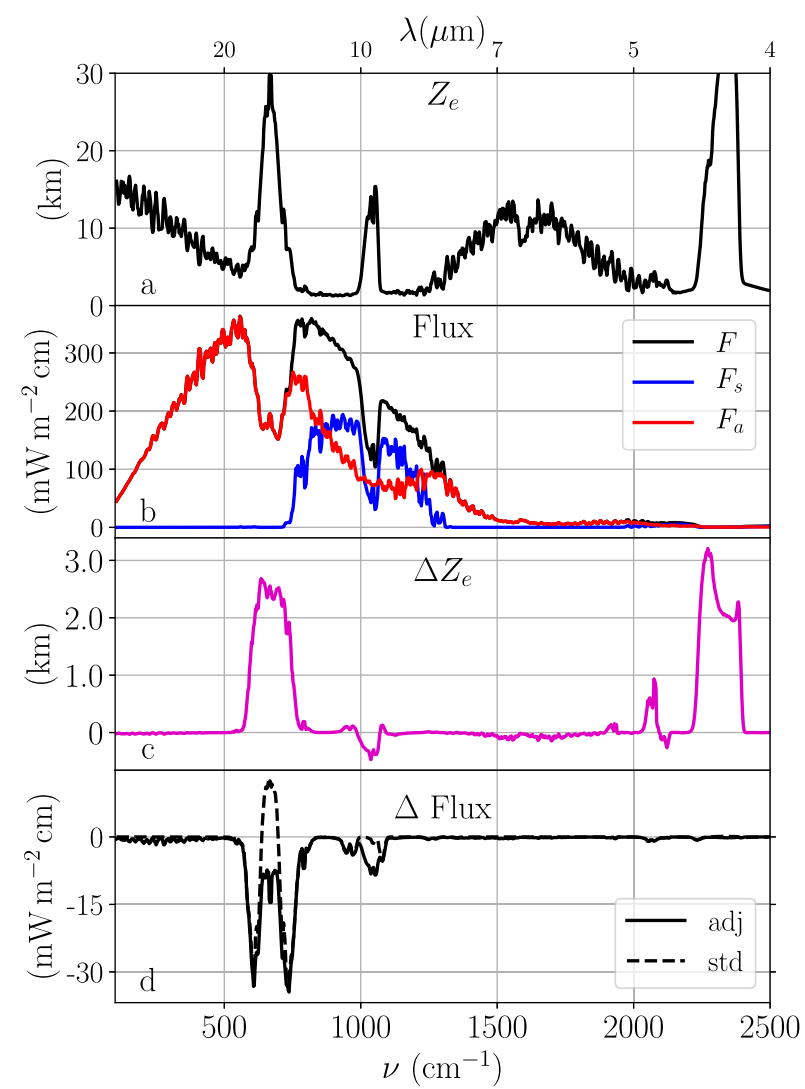

FIG. 14. For the MLS atmospheric profile including the stratosphere, (a) emission height $Z_{e}$ and (b) radiative flux at the TOA in total (black), emitted by the surface (blue), and emitted by the atmosphere (red). Also shown is change in (c) emission height $\left(\Delta Z_{e}\right.$, magenta) and (d) the flux at the TOA if the temperature in the stratosphere is held fixed (dashed line) or is adjusted (solid line). The abscissa is given in wavenumber $\left(\mathrm{cm}^{-1}\right)$ at the bottom and in wavelength $(\mu \mathrm{m})$ at the top. Variables are smoothed on a $10-\mathrm{cm}^{-1}$ spectral interval.

higher than the tropopause but the stratospheric adjustment is considered (Fig. 14d, continuous line). At the first order, the interpretation of the results we obtained with an atmosphere reduced to the troposphere can be extended to a full atmosphere where the temperature of the stratosphere is adjusted. However, the adjustment of the stratosphere also impacts the emission by other gases: $\mathrm{H}_{2} \mathrm{O}$ for wavenumbers lower than $500 \mathrm{~cm}^{-1}$ and ozone near $1050 \mathrm{~cm}^{-1}$.

\section{Summary and conclusions}

In this article we presented a framework that allows us to make a direct and precise link between the basic radiative transfer equations in the atmosphere on one hand, and the concept of emission height on the other hand. This allowed us to quantify how much a change in the greenhouse effect originates from a change in the emission height and how much originates from a change in the absorptivity of the atmosphere (i.e., the absorption over the entire height of the atmosphere).

The fact that a saturation of the absorptivity of the atmosphere leads to a saturation of the greenhouse effect is directly related to the hypothesis of an isothermal atmosphere. When this simplification is removed and the decrease of temperature with altitude is considered, as is the case in the troposphere, the greenhouse effect can continue to increase even if the absorptivity of the atmosphere is saturated.

The fundamental difference between our approach and other approaches such as the "bulk emission temperature" (Benestad 2017) or the "brightness temperature" commonly used in remote sensing, is that we split the radiation leaving the atmosphere toward space into two terms: the radiation that has been emitted by the surface [termed "surface transmitted irradiance" in Costa and Shine (2012)] and the radiation that has been emitted by the atmosphere. The fraction between these two terms is directly driven by the absorptivity of the atmosphere [Eq. (8a)]. When the absorptivity is zero, the total flux leaving the atmosphere originates from radiation emitted by the surface, and the atmosphere has no radiative impact. When the absorptivity is close to 1 (i.e., when the total optical thickness of the atmosphere is larger than about 4), the opposite situation happens: the total flux leaving the atmosphere has been emitted by the atmosphere, the surface does not have any direct radiative impact on the flux leaving the atmosphere, and increasing the optical thickness does not have any influence on the ratio between these two terms anymore. However, this does not mean that the greenhouse effect does not change. Increasing the optical thickness increases the mean emission height and if the atmosphere is not isothermal, a change in emission height translates in a change in outgoing radiative flux.

For an increase in $\mathrm{CO}_{2}$ concentration above its preindustrial value, the increase of the greenhouse effect is primarily due (by about $90 \%$ ) to the change in emission height. In spectral regions that actually contribute to the radiative forcing, the increase in emission height is about $1 \mathrm{~km}$ for a doubling of the $\mathrm{CO}_{2}$ concentration. As the mean emission height is about $6 \mathrm{~km}$ (i.e., above where most of the mass of water vapor is located), the radiative effect of this change of emission height is weakly affected by the water vapor amount. This explains why the increase of the greenhouse effect when $\mathrm{CO}_{2}$ increases is weakly dependent on the $\mathrm{H}_{2} \mathrm{O}$ amount (Fig. 1b), in contrast with the broadband absorptivity. The change in emission height will be of comparable magnitude for any other well-mixed absorbing gases in the spectral domains where the absorptivity is saturated. For an increase of water vapor, the change in absorptivity plays a more important 
role (about $40 \%$ ) but the change in emission height is still about $60 \%$. Indeed, away from the atmospheric window, the absorptivity by water vapor becomes saturated and the change in emission height becomes therefore dominant.

The emission height depends on both the temperature profile and the optical properties [Eqs. (14) and (11)]. We showed that the classical assumption that the emission height is close to the altitude where the optical thickness between this altitude and the top of the atmosphere is equal to one is valid only for atmospheres that are optically thick enough $\left(\tau_{s}>4\right)$. For optically thin atmospheres or with and optical thickness close to one, this assumption is not valid and leads to an underestimation of the emission height.

Considering the real temperature vertical profile in the whole atmosphere makes simplified analysis of the greenhouse effect a priori difficult. However, this complexity is essentially eliminated when considering the adjustment of the stratospheric temperature. This had long been shown when considering global fluxes. Here, we have shown that this is also the case when looking at the change in spectral fluxes and emission altitude, and therefore that it is legitimate to replace the vertical profile of the entire atmosphere by the vertical profile of the troposphere alone, for simplified thinking.

Acknowledgments. We thank the reviewers and the editor for their comments and the many valuable suggestions they made that helped to improve the paper, and we thank Audine Laurian for helping to edit the English. This research was initiated during the internships of Mélodie Trolliet, Thomas Gossot, and Cindy Vida. This work was partially supported by the European FP7 IS-ENES2 project (Grant 312979) and the French ANR project MCG-Rad (18-CE46-001203). The Kspectrum and HR_PPart radiative codes are available online (https://www.meso-star.com/en/) under entry "Atmospheric Radiative Transfer."

\section{APPENDIX A}

\section{Analytical Expression for the Idealized Atmosphere}

In this section we take advantage of the assumption of the idealized atmosphere (section 2) and in particular that the spectral Planck function increases linearly with pressure [Eq. (4)].

\section{a. Outgoing radiance $\mathscr{F}$ at the tropopause}

For the idealized atmosphere, Eq. (8b) can be integrated analytically, and one obtains (after an integration by parts)

$$
\mathcal{B}_{e}=\mathcal{B}\left(P_{s}\right)+\left[\mathcal{B}\left(P_{t}\right)-\mathcal{B}\left(P_{s}\right)\right]\left(\frac{1}{1-e^{-\tau_{s}}}-\frac{1}{\tau_{s}}\right)
$$

The outgoing radiance $\mathscr{F}$ at the tropopause is [Eq. (6)]

$$
\mathscr{F}=\mathcal{B}_{s} e^{-\tau_{s}}+\left(1-e^{-\tau_{s}}\right) \mathcal{B}_{e} .
$$

If the atmosphere is optically very thin $\left(\tau_{s} \ll 1\right)$, one may obtain ${ }^{\mathrm{A} 1}$ that $\mathcal{B}_{e} \approx\left[\mathcal{B}\left(P_{t}\right)+\mathcal{B}\left(P_{s}\right)\right] / 2$ and therefore $\mathscr{F} \approx \mathcal{B}_{s}+\tau_{s}\left(\mathcal{B}_{e}-\mathcal{B}_{s}\right)$. The outgoing radiance is the same for the isothermal and nonisothermal atmosphere, it is equal to the radiance $\mathcal{B}_{s}$ emitted by the surface when the atmosphere is perfectly transparent $\left(\tau_{s}=0\right)$, and then decreases linearly with $\tau_{s}$ when the latter increases. In contrast, if the atmosphere is optically very thick $\left(\tau_{s} \gg 1\right)$, $\mathscr{F} \approx \mathcal{B}_{e} \approx \mathcal{B}\left(P_{t}\right)$, the outgoing radiance is equal to the radiance emitted by a blackbody, the temperature of which is that at the tropopause.

\section{b. Weight $\omega(P)$}

The term $\omega(P)$ is the normalized weighting function to compute the equivalent blackbody emission of the atmosphere [Eq. (8b)]. Note that according to Eq. (8c) $\int_{P_{t}}^{P_{s}} \omega(P) d P=1 ;-\omega(P)$ can be interpreted as a probability density function. For the idealized atmosphere, and according to Eqs. (5), (7), and (8c),

$$
\omega(P)=\frac{1}{1-\mathcal{T}_{s}}\left(\frac{-k f}{g}\right) e^{-k f\left(P-P_{t}\right) / g} .
$$

If the atmosphere is optically thin, $\tau_{s}=k f\left(P_{s}-P_{t}\right) / g \ll 1$, and after a first-order Taylor expansion one obtains

$$
\omega(P) \approx \frac{-1}{P_{s}-P_{t}} .
$$

If the atmosphere is optically thin, the weight $\omega$ is constant along the vertical.

If the atmosphere is optically thick, $\tau_{s}=\kappa\left(P_{s}-P_{t}\right) \gg 1$, $\mathcal{T}_{s} \approx 0$, and, according to Eq. (A3), $\omega \approx 0$ in the lower troposphere and $\omega \approx(-k f / g)$ close to the top of the atmosphere.

\section{APPENDIX B}

\section{Difference in TOA Flux when Changing the Atmospheric Absorption}

The difference of the flux at the TOA for two atmospheres that only differ by their absorbing gases can be written using Eqs. (21) and (20b) as

\footnotetext{
${ }^{\mathrm{A} 1} \mathrm{~A}$ second-order Taylor development is required for $1 /\left(1-e^{-\tau_{s}}\right)$.
} 


$$
\begin{aligned}
F_{2}-F_{1}= & \left(\hat{\mathcal{T}}_{s, 2}-\hat{\mathcal{T}}_{s, 1}\right) B_{s}+\left(1-\hat{\mathcal{T}}_{s, 2}\right) \int_{P_{t}}^{P_{s}} \omega_{2}(P) B(P) d P \\
& -\left(1-\hat{\mathcal{T}}_{s, 1}\right) \int_{P_{t}}^{P_{s}} \omega_{1}(P) B(P) d P
\end{aligned}
$$

which can be written as

$$
\begin{aligned}
F_{2}-F_{1}= & \left(\hat{\mathcal{T}}_{s, 2}-\hat{\mathcal{T}}_{s, 1}\right) B_{s} \\
& +\left(1-\hat{\mathcal{T}}_{s, 2}\right) \int_{P_{t}}^{P_{s}}\left[\omega_{2}(P)-\omega_{1}(P)\right] B(P) d P \\
& -\left(\hat{\mathcal{T}}_{s, 2}-\hat{\mathcal{T}}_{s, 1}\right) \int_{P_{t}}^{P_{s}} \omega_{1}(P) B(P) d P
\end{aligned}
$$

and finally as

$$
\begin{aligned}
F_{2}-F_{1}= & \left(\hat{\mathcal{T}}_{s, 2}-\hat{\mathcal{T}}_{s, 1}\right)\left(B_{s}-B_{e, 1}\right) \\
& +\left(1-\hat{\mathcal{T}}_{s, 2}\right)\left(B_{e, 2}-B_{e, 1}\right) .
\end{aligned}
$$

\section{REFERENCES}

Anderson, G. P., S. A. Clough, F. X. Kneizys, J. H. Chetwynd, and E. P. Shettle, 1986: AFGL atmospheric constituent profiles $(0-120 \mathrm{~km})$. Air Force Geophysics Laboratory Tech. Rep. AFGL-TR-86-011, 46 pp.

Ångström, K., 1900: Ueber die Bedeutung des Wasserdampfes und der Kohlensäure bei der Absorptionder Erdatmosphäre (On the importance of water vapor and carbonic acid in the absorption of Earth's atmosphere). Ann. Phys., 308, 720-732, https://doi.org/10.1002/andp.19003081208.

Archer, D., 2011: Global Warming: Understanding the Forecast. Wiley-Blackwell, $212 \mathrm{pp}$.

Arrhenius, S., 1896: On the influence of carbonic acid in the air upon the temperature of the ground. London Edinburgh Dublin Philos. Mag. J. Sci., 41, 237-276, https://doi.org/ 10.1080/14786449608620846.

Augustsson, T., and V. Ramanathan, 1977: A radiative-convective model study of the $\mathrm{CO}_{2}$ climate problem. J. Atmos. Sci., 34, 448-451, https://doi.org/10.1175/1520-0469(1977)034<0448: ARCMSO $>2.0 . \mathrm{CO} ; 2$.

Benestad, R., 2017: A mental picture of the greenhouse effect. Theor. Appl. Climatol., 128, 679-688, https://doi.org/10.1007/ s00704-016-1732-y.

Charlock, T. P., 1984: $\mathrm{CO}_{2}$ induced climatic change and spectral variations in the outgoing terrestrial infrared radiation. Tellus, 36B, 139-148, https://doi.org/10.3402/TELLUSB.V36I3.14884.

Cherkaoui , M., J.-L. Dufresne, R. Fournier, J.-Y. Grandpeix, and A. Lahellec, 1996: Monte Carlo simulation of radiation in gases with a narrow-band model and a net-exchange formulation. ASME J. Heat Transfer, 118, 401-407, https://doi.org/ $10.1115 / 1.2825858$

Cheruy, F., N. Scott, R. Armante, B. Tournier, and A. Chedin, 1995: Contribution to the development of radiative transfer models for high spectral resolution observations in the infrared. J. Quant. Spectrosc. Radiat. Transfer, 53, 597-611, https:// doi.org/10.1016/0022-4073(95)00026-H.

Clough, S., F. Kneizys, and R. Davies, 1989: Line shape and the water vapor continuum. Atmos. Res., 23, 229-241, https:// doi.org/10.1016/0169-8095(89)90020-3.

_ , and M. J. Iacono, 1995: Line-by-line calculation of atmospheric fluxes and cooling rates: 2. Application to carbon dioxide, ozone, methane, nitrous oxide and the halocarbons. J. Geophys. Res., 100, 16519-16 535, https://doi.org/10.1029/95JD01386.

Collins, W. D., and Coauthors, 2006: Radiative forcing by wellmixed greenhouse gases: Estimates from climate models in the Intergovernmental Panel on Climate Change (IPCC) Fourth Assessment Report (AR4). J. Geophys. Res., 111, D14317, https://doi.org/10.1029/2005JD006713.

Costa, S. M. S., and K. P. Shine, 2012: Outgoing longwave radiation due to directly transmitted surface emission. J. Atmos. Sci., 69, 1865-1870, https://doi.org/10.1175/JAS-D-11-0248.1.

Dufresne, J.-L., R. Fournier, C. Hourdin, and F. Hourdin, 2005: Net exchange reformulation of radiative transfer in the $\mathrm{CO}_{2}$ $15-\mu \mathrm{m}$ band on Mars. J. Atmos. Sci., 62, 3303-3319, https:// doi.org/10.1175/JAS3537.1.

Ellingson, R. G., J. Ellis, and S. Fels, 1991: The intercomparison of radiation codes used in climate models: Long wave results. J. Geophys. Res., 96, 8929-8953, https://doi.org/10.1029/90JD01450.

Eymet, V., C. Coustet, and B. Piaud, 2016: kspectrum: An opensource code for high-resolution molecular absorption spectra production. J. Phys. Conf. Ser., 676, 012005, https://doi.org/ 10.1088/1742-6596/676/1/012005.

Forster, P., and Coauthors, 2007: Changes in atmospheric constituents and in radiative forcing. Climate Change 2007: The Scientific Basis, S. Solomon et al., Eds., Cambridge University Press, 129-234.

Fourier, J.-B. J., 1824: Remarques générales sur les températures du globe terrestre et des espaces planétaires (General remarks on the temperatures of the terrestrial globe and planetary spaces). Ann. Chim. Phys., 27, 136-167.

, 1837: General remarks on the temperature of the terrestrial globe and the planetary spaces. Amer. J. Sci., 32 (1), 1-20.

Green, J. S. A., 1967: Division of radiative streams into internal transfer and cooling to space. Quart. J. Roy. Meteor. Soc., 93, 371-372, https://doi.org/10.1002/qj.49709339710.

Hansen, J., D. Johnson, A. Lacis, S. Lebedeff, P. Lee, D. Rind, and G. Russell, 1981: Climate impact of increasing atmospheric carbon dioxide. Science, 213, 957-966, https://doi.org/10.1126/ science.213.4511.957.

- M. Sato, and R. Ruedy, 1997: Radiative forcing and climate response. J. Geophys. Res., 102, 6831-6864, https://doi.org/ 10.1029/96JD03436.

Harries, J. E., H. E. Brindley, P. J. Sagoo, and R. J. Bantges, 2001: Increases in greenhouse forcing inferred from the outgoing longwave radiation spectra of the Earth in 1970 and 1997. Nature, 410, 355-357, https://doi.org/10.1038/35066553.

Held, I. M., and B. J. Soden, 2000: Water vapour feedback and global warming. Annu. Rev. Energy Environ., 25, 441-475, https://doi.org/10.1146/annurev.energy.25.1.441.

Huang, Y., 2013: A simulated climatology of spectrally decomposed atmospheric infrared radiation. J. Climate, 26, 17021715, https://doi.org/10.1175/JCLI-D-12-00438.1. , and M. Bani Shahabadi, 2014: Why logarithmic? A note on the dependence of radiative forcing on gas concentration. J. Geophys. Res. Atmos., 119, 13 683-13 689, https://doi.org/ 10.1002/2014JD022466. 
Kiehl, J. T., 1983: Satellite detection of effects due to increased atmospheric carbon dioxide. Science, 222, 504-506, https:// doi.org/10.1126/science.222.4623.504.

— , and V. Ramanathan, 1983: $\mathrm{CO}_{2}$ radiative parameterization used in climate models: Comparison with narrow band models and with laboratory data. J. Geophys. Res., 88, 5191-5202, https://doi.org/10.1029/JC088iC09p05191.

McClatchey, R. A., R. W. Fenn, J. Selby, and J. Garing, 1972: Optical properties of the atmosphere. Air Force Cambridge Research Laboratory Tech. Rep. AFCRL-72-0497, 3rd ed., 113 pp.

Mlawer, E. J., V. H. Payne, J.-L. Moncet, J. S. Delamere, M. J. Alvarado, and D. C. Tobin, 2012: Development and recent evaluation of the MT_CKD model of continuum absorption. Philos. Trans. Roy. Soc., 370A, 2520-2556, https://doi.org/ 10.1098/rsta.2011.0295.

Mlynczak, M. G., and Coauthors, 2016: The spectroscopic foundation of radiative forcing of climate by carbon dioxide. Geophys. Res. Lett., 43, 5318-5325, https://doi.org/10.1002/ 2016GL068837.

Pierrehumbert, R. T., 2004: Warming the world: Greenhouse effect: Fourier's concept of planetary energy balance is still relevant today. Nature, 432, 677, https://doi.org/10.1038/432677a.

, 2010: Principles of Planetary Climate. Cambridge University Press, $652 \mathrm{pp}$.

2011: Infrared radiation and planetary temperature. Phys. Today, 64, 33-38, https://doi.org/10.1063/1.3541943.
Pincus, R., and Coauthors, 2015: Radiative flux and forcing parameterization error in aerosol-free clear skies. Geophys. Res. Lett., 42, 5485-5492, https://doi.org/10.1002/2015GL064291.

Rothman, L. S., and Coauthors, 2013: The HITRAN2012 molecular spectroscopic database. J. Quant. Spectrosc. Radiat. Transfer, 130, 4-50, https://doi.org/10.1016/j.jqsrt.2013.07.002.

Schwarzkopf, M. D., and S. Fels, 1991: The simplified exchange method revisited: An accurate, rapid method for computation of infrared cooling rates and fluxes. J. Geophys. Res., 96, 90759096, https://doi.org/10.1029/89JD01598.

Scott, N. A., and A. Chedin, 1981: A fast line-by-line method for atmospheric absorption computations: The automatized atmospheric absorption atlas. J. Appl. Meteor., 20, 802-812, https:// doi.org/10.1175/1520-0450(1981)020<0802:AFLBLM>2.0.CO;2.

Shine, K., Y. Fouquart, V. Ramaswamy, S. Solomon, and J. Srinivasan, 1995: Radiative forcing. Climate Change 1994: Radiative Forcing of Climate Change and Evaluation of the IPCC IS92 Emission Scenarios, J. Houghton et al., Eds., Cambridge University Press, 35-71.

Stuber, N., R. Sausen, and M. Ponater, 2001: Stratosphere adjusted radiative forcing calculations in a comprehensive climate model. Theor. Appl. Climatol., 68, 125-135, https://doi.org/ 10.1007/s007040170041.

Zhong, W., and J. D. Haigh, 2013: The greenhouse effect and carbon dioxide. Weather, 68, 100-105, https://doi.org/10.1002/ wea.2072. 